\title{
LE COMPORTEMENT DE NIDIFICATION CHEZ OSMIA (OSMIA) CORNUTA LATR. ET OSMIA (OSMIA) RUFA L. (HYMENOPTERA MEGACHILIDAE)*
}

\author{
Das Nestbau- Verhalten von Osmia (Osmia) cornuta Latr. \\ und Osmia (Osmia) rufa L. (Hymenoptera Megachilidae)
}

\author{
Jean-Noël TASEI \\ avec la collaboration technique de Murielle PICART \\ Laboratoire d'Éthologie et d'Écologie des Insectes, I.N.R.A., \\ 86 - Lusignan \\ SUMMARY \\ Nesting Behaviour of Osmia (Osmia) cornuta \\ LATR. AND Osmia (Osmia) rufa L. \\ (Hymenoptera Megachilidae)
}

The nesting behaviour of Osmia cornuta Latr. and Osmia rufa L. has been studied by the author for two consecutive years in Lusignan area.

Both species build their nests in holes of old timber and walls facing North or West. Though the nests are similar the building technics are different : $O$. cornuta doesn't build any " threshold ", $O$. rufa builds one for each cell. The pollen provisions of $O$. cornuta are uniformly damp and the eggs are clear of pollen; the provisions and the eggs of $O$. rufa are powdered with dry pollen.

In greenhouse the osmia provision their nest with pollen of Rosaceae, Cruciferae, Papilionaceae (Trifolium), and Salix. A cell is filled after 30 to 40 foraging trips in 4 to 5 hours. In the nature these two polylectic species which collect pollen on about 10 plants families show oligolectic tendencies : $O$. cornuta collects pollen chiefly on Prunus, $O$. rufa on Quercus and Ranunculus.

* Ce travail constitue une partie d'une thèse de docteur de spécialité soutenue le 25 avril 1972 devant l'Université Paul Sabatier de Toulouse. 


\section{RÉSUME}

L'auteur a étudié pendant deux années consécutives le comportement de nidification chez Osmia cornuta Latr. et Osmia rufa L. dans la région de Lusignan.

Les deux espèces construisent de préférence leurs nids dans des trous de vieilles charpentes et de murs de pierre exposés au Nord et à l'Ouest. Les nids sont assez semblables mais la technique de construction est différente d'une espèce à l'autre : $O$. cornuta ne construit pas de " pas de porte " tandis qu' $O$. rufa en construit généralement un pour chaque cellule. Les cellules d'O. cornuta contiennent une provision de pollen uniformément humectée et un ceuf sans trace de pollen. Les cellules d' $O$. rufa contiennent une provision et un ouf plus ou moins recouverts d'une couche de pollen sec.

En serre les osmies approvisionnent leur nid avec le pollen de Rosaceae, Cruciferae, Papilionaceae (Trifolium) et Salix. Une cellule est approvisionnée en 4 à 5 heures, soit après 30 à 40 voyages de butinage. Dans la nature ces 2 espèces polylectiques récoltant le pollen sur près de 10 familles botaniques manifestent une certaine tendance à l'oligolectie : $O$. cornuta récolte surtout sur Prunus, O. rufa sur Quercus et Ranunculus.

\section{INTRODUCTION}

De nombreux auteurs : Fabre (1913), Ferton (1923), Hardouin (1948), etc. ont été captivés par les mœurs des osmies et leurs ouvrages reflètent la grande variété des comportements de nidification que l'on rencontre chez le genre Osmia. Généralement ces auteurs ont, soit étudié un point très particulier de la nidification d'une espèce (construction, ponte, repérage du nid...), soit fait une revue biologique chez plusieurs espèces.

Deux osmies : $O$. cornuta et $O$. rufa ont éveillé depuis longtemps l'attention des spécialistes (Popovici Baznosanu, 1910; Friese, 1923; Maréchal, 1926; Lecomte, 1963-65; Descy, 1968; Free et Williams, 1970). Dans le présent travail nous avons voulu effectuer une étude comparative détaillée du comportement de nidification chez les 2 espèces.

$O$. cornuta et $O$. rufa sont communes en Europe. La première espèce ne se rencontre pas dans les pays nordiques; elle devient abondante dans le Sud de l'Europe; par contre $O$. rufa a une aire de répartition beaucoup plus vaste. En France les 2 espèces se rencontrent aussi bien dans la région parisienne qu'en Provence.

Notre étude s'est poursuivie pendant 2 ans à Lusignan ${ }^{1}$. Nous avons observé les osmies dans leur milieu naturel sans négliger les possibilités offertes par l'élevage en enceinte fermée. Le comportement de construction et d'approvisionnement du nid ainsi que le comportement de butinage ont pu ainsi être analysés avec précision.

1. Ville située à $25 \mathrm{~km}$ au Sud-Ouest de Poitiers (Vienne). 


\section{MATÉRIEL ET METHODES}

\section{1. - Le dispositif d'observation dans la nature}

Nous avons utilisé le même dispositif que dans une étude précédente (TASEI, 1972). Il s'agit de 55 nichoirs en bois contenant des tubes en carton et des cannes de Provence fendues en 2 parties longitudinales. Les diamètres des cavités sont : 4,5 et $8 \mathrm{~mm}$. Les nichoirs sont accrochés à la fin de l'hiver contre des parois de bâtiments, contre des arbres, etc. dans divers hameaux et fermes des environs de Lusignan.

Une fois par semaine on retire les nids qui sont entièrement construits ou qui sont abandonnés et on les remplace par des tubes vides.

\section{2. - L'élevage en serre}

Certaines observations concernant l'activité de construction et l'approvisionnement du nid ne peuvent être effectuées qu'en enceinte fermée. Trois femelles d'O. cornuta et quatre femelles d'O. rufa sont introduites dans un compartiment de serre vitrée pouvant être ombrée et aérée. Les femelles sont capturées alors qu'elles commencent à nidifier dans la nature. Une cinquième femelle d' $O$. rufa a nidifié en même temps que les autres après avoir émergé en laboratoire en compagnie de quelques mâles.

Afin de connaître le comportement des femelles à l'intérieur du nid on retire, au cours de la période d'activité, tous les tubes de canne de Provence et on les remplace par des tubes de verre fermés à une extrémité. Les tubes ont 5 ou $6,5 \mathrm{~cm}$ de long et 9 ou $12 \mathrm{~mm}$ de diamètre. Ce dispositif employé avec succès par Fabre (1913), SAz (1929), Lozinskr (1936) et Hartman (1944) pour l'étude de la biologie de plusieurs espèces d'Osmies n'a permis que peu de nidifications. En effet une seule femelle d'O. rufa a accepté de construire dans de telles cavités.

Enfin au cours de la construction et de l'approvisionnement des cellules nous avons troublé le comportement d'une femelle d'O. cornuta et de deux femelles d'O. rufa. Cette étude expérimentale consiste à achever le travail de la femelle de telle sorte qu'elle ne puisse pas accomplir une partie de la séquence de nidification, ou qu'elle soit gênée pour l'accomplir, ou bien on détruit la même partie de la cellule à divers moments de la séquence ou bien on déplace le nid, etc.

\section{3. - Techniques de détermination qualitative et quantitative des pollens récoltés dans la nature}

A chaque visite hebdomadaire des " pièges-nichoirs " on prélève avec une pointe de scapel un échantillon de pollen dans la première cellule et généralement aussi dans la dernière cellule construite dans les nids operculés. Le prélèvement se fait dans le sens dela longueur de la provision de pollen. 31 cellules d' $O$. cornuta et 52 d'O. rufa ont fait l'objet de tels prélèvements. Il est recommandé de monter les échantillons de pollen (méthode Mavnizio et Louveaux, 1965) le plus rapidement possible, car les femelles incorporent à la pâtée pollinique une certaine quantité de sécrétions salivaires qui “ digèrent » le cytoplasme des grains et altèrent la forme générale du pollen. En outre on doit préciser que les méthodes de montage et de détermination employées ici, ne permettent pas l'analyse microscopique des excréments de larve, car les grains de pollen émis hors du tube digestif, sont réduits à l'état de coques froissées et vides non identifiables.

La distinction entre pollens étant parfois très délicate on a recours à un tableau indiquant les dates de début et de fin de floraison des espèces végétales, observées pendant la saison d'étude.

On procède sur chaque préparation à un comptage jusqu'à un total de 250 à 300 grains. Cette opération s'effectue grâce à une série de numérations dans des champs répartis au hasard sur toute la lame. On calcule ensuite les pourcentages des différentes espèces de pollen. Ces chiffres donnent les proportions numériques des pollens récoltés, mais si l'on désire évaluer les proportions volumétriques on doit corriger les nombres de grains de pollen à l'aide de coefficients correctifs comme l'indique le tableau 1.

Nos méthodes d'analyse microscopique des provisions des cellules d'Osmies nous renseignent principalement sur :

- les espèces butinées pour le pollen,

- les dates de récolte des divers pollens,

- l'influence de la localité sur le spectre pollinique de la récolte.

Une question reste posée : y a-t-il proportionnalité entre le temps de butinage sur différentes plantes et les volumes de pollen correspondants? Nous avons effectué à ce sujet des 
TaBleau 1. - Calcul et utilisation d'un coefficient correctif

TABELLE 1. - Berechnung und Anwendung eines Korrektiv-Koeffizienten

\begin{tabular}{|c|c|c|c|}
\hline 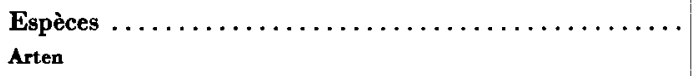 & $n^{\circ} 1$ & $n^{\circ} 2$ & $n^{\circ} 3$ \\
\hline $\begin{array}{l}\text { Nombre de grains } \ldots \ldots \ldots \ldots \ldots \ldots \ldots \ldots \ldots \\
\text { Anzahl d. Pollenkörner. }\end{array}$ & $=n \mathbf{l}$ & $n 2$ & $n 3$ \\
\hline $\begin{array}{l}\text { Diamètre moyen d'un grain } \ldots \ldots \ldots \ldots \ldots \ldots \ldots \ldots \ldots \\
\text { Durchschnittl. Durchmesser eines Pollenkorns. }\end{array}$ & $=d 1$ & $d 2$ & $d 3$ \\
\hline $\begin{array}{l}\text { Volume moyen d'un grain } \ldots \ldots \ldots \ldots \ldots \ldots \ldots \ldots \ldots \ldots \\
\text { Durchschnittl. Inhalt eines Pollenkorns. }\end{array}$ & $=\mathrm{K} d_{1}^{3}$ & $\mathbf{K} d_{2}^{3}$ & $\mathrm{~K} d_{3}^{3}$ \\
\hline $\begin{array}{l}Q \text { coefficient correctif volumétrique } \ldots \ldots \cdots \cdots \\
\text { Volumetrischer } Q \text { Korrektiv-Koeffizient. }\end{array}$ & $=\mathbf{Q}_{\mathbf{I}}=\mathbf{1}$ & $\mathrm{Q}_{2}=\frac{d_{2}^{3}}{d_{1}^{3}}$ & $\mathrm{Q}_{3}=\frac{d_{3}^{3}}{d_{1}^{s}}$ \\
\hline $\begin{array}{l}\text { Volume (proportions) } \ldots \ldots \ldots \ldots \ldots \ldots \ldots \ldots \ldots \ldots \ldots \cdots \\
\text { Inhalt (Proportionen). }\end{array}$ & $=n_{1} \times Q_{1}$ & $n_{2} \times Q_{2}$ & $n_{3} \times Q_{3}$ \\
\hline
\end{tabular}

observations préliminaires : chronométrages en serre du butinage sur plusieurs espèces de plantes et analyses microscopiques des chargements de pollens correspondants. Ces observations portant sur 3 espèces d'Osmies (O. cornuta, $O$. rufa, 0 . coerulescens L.) ne sont pas suffisamment nombreuses et précises pour confirmer l'hypothèse de proportionnalité. Il serait risqué dans certains cas de dire qu'une plante fournit un volume de pollen plus élevé qu'une autre quand elle est butinée plus de temps. Le facteur temps de butinage est probablement le facteur principal de l'importance du volume de pollen accumulé dans les cellules, mais il n'est pas le seul. Le mode de présentation du pollen ne doit pas avoir un rôle négligeable : par exemple la récolte du pollen de châtons est certainement plus rapide que la récolte du pollen de fleurs papilionacées.

\section{RÉSULTATS}

\section{1. - L'activitê générale}

a) Les périodes de vol

La période de vol des femelles s'étend à peu près sur 2 mois pour les 2 espèces : début avril à début juin pour $O$. cornuta, fin avril à fin juin pour O. rufa. La période de vol des mâles est beaucoup plus courte que celle des femelles : environ 15 jours (fig. 1). Les dénombrements de femelles en activité dans les nichoirs révèlent qu'en 1970 et 1971 O. Rufa est plus abondante qu'O. cornuta.

En $1971, O$. cornuta a construit et approvisionné des cellules du 7 avril au 26 mai et $O$. rufa du 14 avril au 9 juin. La comparaison de la fig. 1 et du tableau 2 


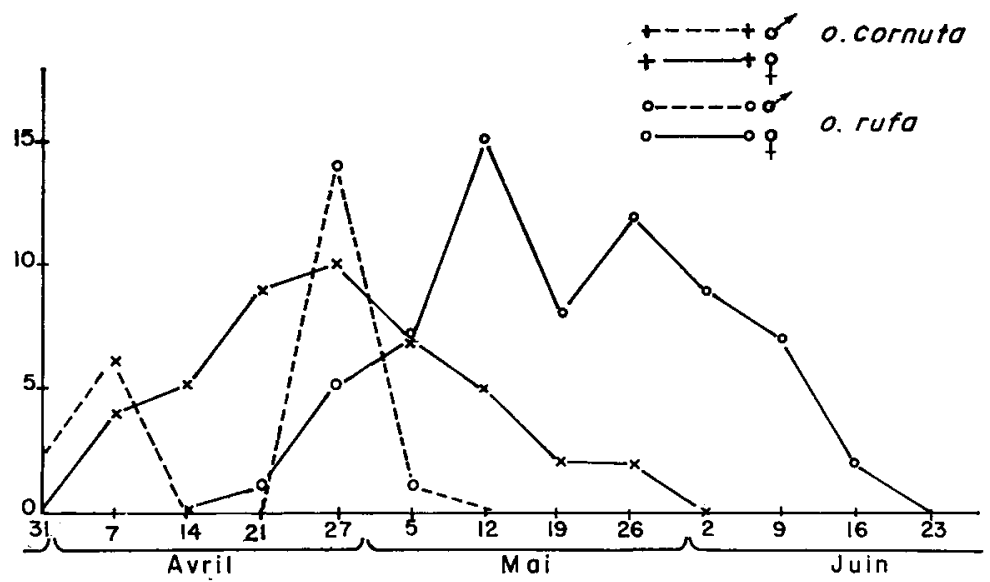

Fig. 1. - Présence d'adules d'O. cornuta et d'O. rufa dans les nichoirs selon la date. Lusignan 1971. Verticalement $=$ adultes dénombrés dans 55 nichoirs.

ABB. 1. - Zeitlich festgestelle Adulte von 0 . cornuta und 0 . rufa in den Nistanlagen. Lusignan 1971. Ordinate $=$ In 55 Nistanlagen gezählte Adulte.

montre que le nombre de cellules n'est pas toujours proportionnel au nombre de femelles actives. Ces discordances peuvent être expliquées par l'influence des conditions climatiques défavorables survenant entre 2 visites de nichoirs. Par ailleurs on remarque que dans les mêmes conditions, une femelle d'O. rufa construit en moyenne plus rapidement qu'O. cornuta.

Tableau 2. - Activité de construction dans les conditions naturelles chez 0 . cornuta et 0 . rufa dans les " piège-nichoir 》. Lusignan 1971

TABELle 2: - Bautätigkeit von 0 . cornuta und 0 . rufa unter natürlichen Bedingungen in « Fallennestern ». Lusignan 1971

\begin{tabular}{|c|c|c|c|c|c|c|c|c|c|}
\hline $\begin{array}{l}\text { Date } \\
\text { Datum }\end{array}$ & $\begin{array}{c}7-4 \\
\text { au } 14 / 4\end{array}$ & $\begin{array}{c}14 / 4 \\
\text { au } 21 / 4\end{array}$ & $\left|\begin{array}{c}21 / 4 \\
\text { au } 28 / 4\end{array}\right|$ & $\begin{array}{c}28 / 4 \\
\text { au } 5 / 5\end{array}$ & $\begin{array}{c}5 / 5 \\
\text { au } 12 / 5\end{array}$ & $\begin{array}{c}12 / 5 \\
\text { au } 19 / 5\end{array}$ & $\begin{array}{c}19 / 5 \\
\text { au } 26 / 5\end{array}$ & $\begin{array}{c}26 / 5 \\
\text { au } 2 / 6\end{array}$ & $\begin{array}{l}2 / 6 \\
\operatorname{au} 9 / 6\end{array}$ \\
\hline $\begin{array}{l}\text { Nombre de cellules } \\
\text { Zahl d. Zellen }\end{array}$ & & & & & & & & & \\
\hline -O. cornuta & 3 & 8 & 6 & 7 & 2 & 2 & 9 & - & - \\
\hline 一. & - & 2 & - & 17 & 50 & 20 & 9 & 5 & 3 \\
\hline
\end{tabular}

\section{b) L'activité en serre}

L'activité en serre est résumée dans le tableau 3. Le temps de latence qui est nécessaire à la reprise de l'activité de construction après la capture, ainsi que la longévité des femelles sont assez variables. La durée de nidification ne comprend pas la dernière période plus ou moins longue, pendant laquelle les 
TABLEAU 3. - Nidification de 8 Osmies en serre à Lusignan. 1970-1971

TabelLe 3. - Nestbau von 8 Osmien im Gewächshaus. Lusignan 1970-1971

\begin{tabular}{|c|c|c|c|c|c|c|}
\hline & 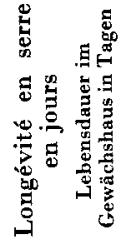 & 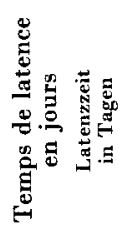 & 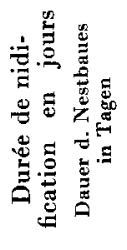 & 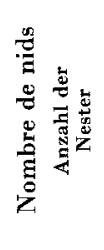 & 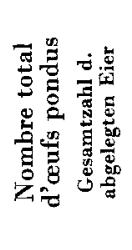 & 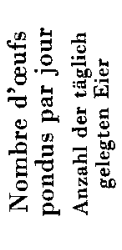 \\
\hline 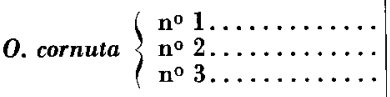 & $\begin{array}{l}18 \\
30 \\
19\end{array}$ & $\begin{array}{r}10 \\
5 \\
9\end{array}$ & $\begin{array}{r}8 \\
21 \\
10\end{array}$ & $\begin{array}{l}6 \\
6 \\
3\end{array}$ & $\begin{array}{r}13 \\
10 \\
6\end{array}$ & $\begin{array}{l}1,6 \\
0,5 \\
0,7\end{array}$ \\
\hline 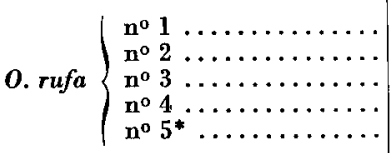 & $\begin{array}{r}26 \\
23 \\
58 \\
2 \\
57\end{array}$ & $\begin{array}{r}15 \\
7 \\
3 \\
31\end{array}$ & $\begin{array}{r}11 \\
16 \\
48 \\
0 \\
26\end{array}$ & $\begin{array}{l}6 \\
8 \\
8 \\
0 \\
3\end{array}$ & $\begin{array}{r}19 \\
24 \\
14 \\
0 \\
10\end{array}$ & $\begin{array}{l}1,7 \\
3,0 \\
0,3 \\
0 \\
0,4\end{array}$ \\
\hline
\end{tabular}

* O. rufa no 5 a été obtenue en laboratoire.

o. rufa Nr. 5 wurde im Labor gezogen.

femelles senescentes construisent anarchiquement sans pondre. La fécondité est légèrement sous estimée puisque les osmies ont déjà commencé à pondre à l'extérieur, cependant elle est en moyenne proche de celle qui est estimée par les auteurs.

En serre les Osmies butinent près de 14 heures par jour lorsque le ciel est clair. O. cornuta commence à visiter les fleurs lorsque la température dépasse $11^{\circ} \mathrm{C}$, elle cesse toute activité au coucher du soleil même si la température est élevée. $O$. rufa a besoin d'une température supérieure pour voler. Les deux espèces passent la nuit à l'intérieur de leur nid la tête ou l'abdomen touchant la dernière cellule construite. Pendant la journée, au cours de l'approvisionnement des cellules de pollen, ou bien au cours de la construction, on observe des poses de femelles qui prennent des « bains de soleil ». Lorsqu'une femelle meurt il est très rare de la retrouver dans son nid.

\section{2. - L'architecture du nid}

Chez les deux espèces les nidifications en tube de bambou se présentent comme l'indique la fig. 2 : la cavité est compartimentée par des cloisons de boue durcie. Nous appelons bouchon initial la première cloison souvent construite à l'extrémité du tube et bouchon terminal la dernière cloison construite à l'autre extrémité. A l'intérieur du tube les cloisons délimitent des cellules vides ou habitées. Presque toujours il y a une cellule vide, ou vestibule, à l'entrée du nid. 


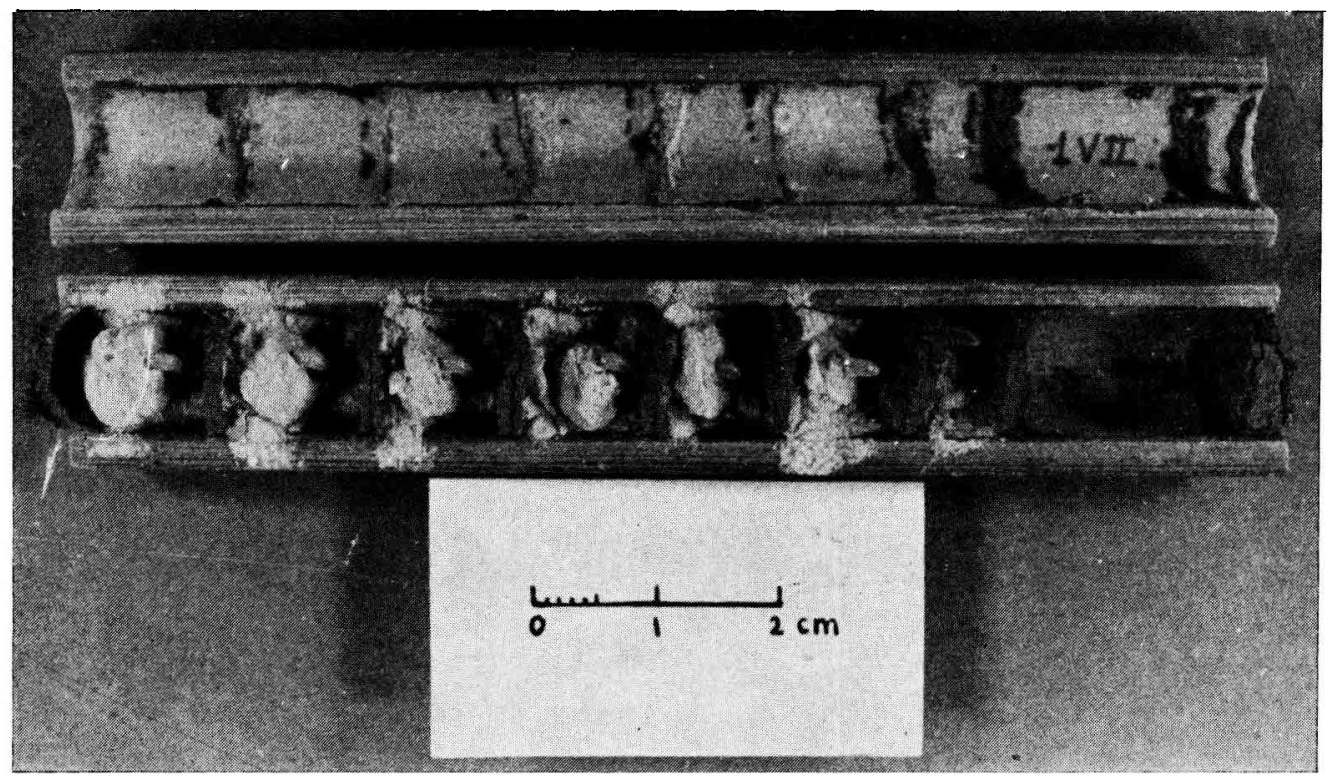

FIG. 2. - IViả ảOsmia rûa ảans une canne de Provence. Le bouchon terminal est à droite.

Aвв. 2. - Nest von Osmia rufa in einem Rohrstengel. Der Verschlusspfropfen befindet sich rechts

Chez $O$. cornuta et $O$. rufa les cloisons ont leur concavité tournée vers l'entrée du nid, leur épaisseur moyenne est de 1 à $2 \mathrm{~mm}$, mais elle augmente à l'endroit de leur jonction avec les parois du tube. Les bouchons terminaux sont toujours plus épais que les cloisons : 6 à $7 \mathrm{~mm}$ chez 0 . cornuta, 1 à $8 \mathrm{~mm}$ chez $O$. rufa. Les vestibules ont des longueurs très variables : 3 à $18 \mathrm{~mm}$ chez $O$. cornuta et 2 à $52 \mathrm{~mm}$ chez $O$. rufa. Les cellules habitées ont des longueurs beaucoup plus constantes. Le tableau $n^{\circ} 4$ indique les fréquences des longueurs des cellules selon le diamètre du tube.

En moyenne les cellules d' $O$. cornuta sont plus longues que celles de $O$. rufa, quelque soit le diamètre utilisé. Lorsque le diamètre augmente $O$. cornuta semble réduire la longueur des cellules (18 à 12,5 mm) alors qu'O. rufa semble conserver à peu près la même longueur $(10-12 \mathrm{~mm})$. Malgré la réduction de la longueur due à l'accroissement du diamètre, le volume moyen des cellules d'O. cornuta est de $225 \mathrm{~mm}^{3}$ et $630 \mathrm{~mm}^{3}$ pour les diamètres de 4 et $8 \mathrm{~mm}$ respectivement. La distribution des longueurs de cellules est beaucoup plus étalée chez $O$. cornuta que chez $O$. rufa. Cette différence de variabilité dans l'architecture sera expliquée plus loin $(\S 3)$. Quelques auteurs font part d'observations complémentaires : Popovici Baznosanu (1909) constate parfois dans des nids des deux espèces la présence $d$ 'une arrière-galerie lorsque le bouchon initial n'est pas construit à l'extrémité du tube, ou bien l'absence de pollen dans certaines cellules. Le même auteur remarque que $50 \%$ des nids, bien que 
TaBLead 4. - Influence du diamètre du tube de nidification sur la longueur et le volume des cellules

TABelle 4. - Einfluss des Durchmessers der Nestbauröhrchen auf Länge u. Volumen d. Zellen

\begin{tabular}{|c|c|c|c|c|c|c|c|c|c|}
\hline \multirow{2}{*}{$\begin{array}{l}\text { Diamètre du tube } \\
\text { en mm } \\
\text { Durchmesser } \\
\text { des Rohres in mm }\end{array}$} & \multicolumn{7}{|c|}{$\begin{array}{l}\text { Longueur des cellules en mm } \\
\text { Länge der Zellen in mm }\end{array}$} & \multirow{2}{*}{\begin{tabular}{|c} 
Longueur \\
moyenne \\
en mm \\
Durchschnittliche \\
Länge in mm
\end{tabular}} & \multirow{2}{*}{$\begin{array}{c}\text { Volume } \\
\text { moyen en mm3 } \\
\text { Durchschnittl. } \\
\text { Inhalt in } \mathrm{mm}^{3}\end{array}$} \\
\hline & $\begin{array}{c}7 \\
\mathbf{a} \\
10\end{array}$ & $\begin{array}{c}10 \\
\text { à } 12\end{array}$ & $\begin{array}{c}12 \\
\text { à } 14\end{array}$ & $\begin{array}{c}14 \\
\text { à } 16\end{array}$ & $\begin{array}{c}16 \\
\text { à } 18\end{array}$ & $\begin{array}{c}18 \\
\text { à } 20\end{array}$ & $\begin{array}{c}20 \\
\text { à } 23\end{array}$ & & \\
\hline $4\left\{\begin{array}{l}\text { O. cornuta } \ldots \ldots \ldots \ldots \\
\text { o. rufa } \ldots \ldots \ldots \ldots\end{array}\right.$ & $\begin{array}{l}1 \\
3\end{array}$ & $\begin{array}{l}1 \\
1\end{array}$ & - & - & $\begin{array}{l}1 \\
-\end{array}$ & $\begin{array}{l}4 \\
-\end{array}$ & $\begin{array}{l}2 \\
-\end{array}$ & $\begin{array}{r}18,0 \\
9,0\end{array}$ & $\begin{array}{l}225 \\
113\end{array}$ \\
\hline $5\left\{\begin{array}{l}\text { O. cornuta } \ldots \\
\text { o. rufa } \ldots . .\end{array}\right.$ & $\begin{array}{c}1 \\
13\end{array}$ & $\begin{array}{c}3 \\
49\end{array}$ & $\begin{array}{c}6 \\
33\end{array}$ & $\begin{array}{l}2 \\
8\end{array}$ & $\begin{array}{l}2 \\
-\end{array}$ & $\begin{array}{l}1 \\
-\end{array}$ & $\begin{array}{l}4 \\
-\end{array}$ & $\begin{array}{l}15,0 \\
11,7\end{array}$ & $\begin{array}{l}425 \\
332\end{array}$ \\
\hline $8\left\{\begin{array}{l}\text { O. cornuta .. } \\
\text { O. rufa .... }\end{array}\right.$ & $\begin{array}{l}3 \\
21\end{array}$ & $\begin{array}{l}9 \\
19\end{array}$ & $\begin{array}{c}5 \\
14\end{array}$ & $\begin{array}{l}5 \\
-\end{array}$ & $\begin{array}{c}4 \\
--\end{array}$ & - & - & $\begin{array}{l}12,5 \\
11,0\end{array}$ & $\begin{array}{l}630 \\
553\end{array}$ \\
\hline 10 à 11 O. rufa $\ldots .$. & 9 & 6 & 1 & - & - & - & - & 9,8 & 846 \\
\hline
\end{tabular}

fondés dans des roseaux de 9 à $26 \mathrm{~cm}$ de long ne possèdent qu'une cellule. Nous avons constaté une meilleure utilisation des cavités lors de nos deux années d'observation (4 à 6 cellules par tube). Descy (1968) a réussi à faire nidifier $O$. rufa dans des tubes de $35 \mathrm{~mm}$ de diamètre et a obtenu, non plus un cloisonnement de la cavité mais la fabrication de pots de boue séchée construits, de préférence dans les angles, et recouverts d'un crépis de boue. Bonelli (1966) a trouvé chez la même espèce des cellules proches de celles observées par Descy, ayant des parois latérales et un toit de boue séchée.

Plusieurs auteurs remarquent que les cellules proches de l'entrée du nid sont plus courtes que celles du fond. Nous avons noté cette architecture dans certains nids chez les deux espèces. Ces cellules, proches de la sortie abritent toujours des mâles.

\section{3. - Le comportement de construction}

$O$. cornuta commençant un nid élève une première cloison (bouchon initial) puis accumule un mélange de pollen et de nectar sur lequel elle dépose un œuf, enfin élève une deuxième cloison à quelque distance de l'œuf.

La séquence de nidification d'O. rufa diffère quelque peu : la femelle élève la première cloison, puis délimite la future cellule en construisant un " pas de porte m. Il s'agit de l'ébauche de la deuxième cloison qui a la forme d'un crois- 
sant ou d'un anneau de boue développé à la partie inférieure en lame verticale. Après la construction du “ pas de porte » la femelle confectionne sa provision de pollen, pond, puis finit d'élever la deuxième cloison.

Descy (1968) est le seul auteur à noter comme nous, qu'O. cornuta ne construit pas l'ébauche de la deuxième cloison avant d'approvisionner la cellule. L'édification du “ pas de porte ) est un comportement très répandu dans le genre Osmia : Osmia lignaria Say édifie un ( pas de porte » en boue (Levin, 1966). Osmia georgica Cres. édifie un “ pas de porte » en pulpe de feuilles mâchées (Hartman, 1944). Osmia coerulescens L. édifie “ un pas de porte 》 en pulpe de feuilles de Malva sylvestris (TASEI, 1972).

Ce “ pas de porte " peut être réduit à une simple marque de ciment végétal ou minéral lorsque le diamètre du tube de nidification est trop faible. On peut supposer que la construction du “ pas de porte " permet à $O$. rufa d'établir des cellules de longueur plus constante que dans le cas d'O. cornuta (cf. tableau 4).

Les femelles d'O. cornuta et d'O. rufa recherchent dans la serre ou dans la nature un coin de terre humide, elles grattent le sol avec les pattes I, les pattes II et III servant de support. Les pattes II peuvent effectuer des mouvements très rapides de balayage du sol. Parfois les femelles s'arc-boutent et la terre est malaxée entre la pointe de l'abdomen et les mandibules. Heroin-Déauney (1966) a prouvé grâce à l'emploi de produits radio-actifs, qu'O. cornuta mélange à la boue des sécrétions. Cet auteur estime que des phénomènes enzymatiques interviennent dans le durcissement de la terre des cloisons. Les travaux de maçonneries sont exécutés seulement avec les mandibules. Les cloisons sont

Tableau 5. - Temps de construction chez 0 . cornuta et 0 . rufa en serre. Lusignan 1970

TABelle 5. - Bauzeiten bei O. cornuta und O. rufa im Gewächshaus, Lusignan 1970

\begin{tabular}{|c|c|c|c|c|c|c|}
\hline $\begin{array}{c}\text { Espèce } \\
\text { Art }\end{array}$ & $\begin{array}{c}\text { Construction } \\
\text { Bau }\end{array}$ & $\begin{array}{c}\text { Nombre } \\
\text { de voyages } \\
\text { Zahl d. } \\
\text { Ausflüge }\end{array}$ & $\begin{array}{c}\text { Temps total } \\
\text { en mn/s } \\
\text { Gesamtzeit } \\
\text { in Min-Sek }\end{array}$ & $\begin{array}{l}\text { Temps moyen } \\
\text { passế au nid } \\
\text { en mn } / \mathrm{s} \\
\text { Mittlere irn } \\
\text { Nest verbrachte } \\
\text { Zeit Min-Sek }\end{array}$ & $\begin{array}{l}\text { Temps moyen } \\
\text { passé sur terre } \\
\text { en mn } / \mathrm{s} \\
\text { Mittlere auf d. } \\
\text { Erde verbrachte } \\
\text { Zeit Min-Sek }\end{array}$ & $\begin{array}{l}\text { Prise de nectar } \\
\text { en mn } / \mathrm{s} \\
\text { Nektaraufnahme } \\
\text { in Min-Sek }\end{array}$ \\
\hline \multirow{3}{*}{ O. cornuta } & Cloison & 8 & 18,24 & 0,88 & 0,46 & 2,30 \\
\hline & Cloison & 10 & 35,00 & 一 & - & - \\
\hline & $\begin{array}{l}\text { Bouchon } \\
\text { terminal }\end{array}$ & 28 & 42,35 & 0,71 & 0,19 & 0,25 \\
\hline \multirow{3}{*}{ O. rufa $\ldots \ldots$} & Cloison & $\longrightarrow$ & 16,00 & 一 & 一 & - \\
\hline & Cloison & 11 & 30,55 & 2,07 & 0,33 & 0 \\
\hline & Cloison & 8 & 27,30 & 2,35 & 0,25 & 0,40 \\
\hline
\end{tabular}


construites par des apports de boue étalés de façon concentrique, si bien qu'avant l'operculation définitive de la cellule il reste en son centre un petit orifice. Une cloison se fait en deux temps : d'abord l'operculation, puis l'épaississement de l'opercule. Au cours des allées et venues de la terre au nid, les femelles vont parfois butiner 2 à 3 minutes sur les fleurs dont elles prélèvent uniquement le nectar. Le tableau 5 donne quelques temps de construction chronométrés en serre. Ces résultats prouvent que les temps de construction sont très variables mais du même ordre de grandeur pour les deux espèces. D'autre part le temps passé au nid pour appliquer la boue à la cloison est toujours plus important que celui passé à ramasser la boue. II y a une grande variabilité dans les temps des séjours au nid et des séjours sur la terre : séjour sur la terre de 10 secondes à 3 minutes 10 secondes, séjour au nid de 15 secondes à 6 minutes 35 secondes. (Il s'agit des données extrêmes d'O. cornuta et $O$. rufa).

Lorsque la femelle est vieille, il lui arrive de modifier la séquence de nidification et de construire une série de bouchons terminaux sans qu'il y ait dans les tubes bouchés une seule cellule. Malyshev (1935) rapporte qu'O. rufa vieille peut détruire ses propres cellules ou bien construire des cellules trop grandes ou des cellules vides.

\section{4. - Le choix du site de nidification}

Si on estime avec Free et Williams (1970) que d'une part la population d'abeilles d'un nichoir est représentative de la population locale, et que d'autre part les femelles nidifient de préférence sur les lieux de leur naissance, on peut interpréter les tableaux 6,7 et 8 de la manière suivante : seules les constructions en bois et en pierre abritent des nids naturels d'Osmies; les bâtiments crépis sont peu favorables aux abeilles et les arbres et arbustes pas du tout. Le bois mort (poteaux, charpentes, etc.) semble favoriser la nidification des 2 espèces, tandis que les murs de pierres paraissent favoriser $O$. rufa seule.

TABLeaU 6. - Répartition des nids suivant le support du nichoir. Lusignan 1971

Tabelle 6. - Verteilung der Nester entsprechend der Unterlage der Nistgelegenheit. Lusignan 1971

\begin{tabular}{|c|c|c|c|c|}
\hline $\begin{array}{l}\text { Support } \\
\text { Unterlage }\end{array}$ & $\begin{array}{l}\text { Arbustes } \\
\text { Sträucher }\end{array}$ & $\mid \begin{array}{c}\text { Mur de pierre } \\
\text { Steinmauer }\end{array}$ & $\begin{array}{l}\text { Crépis } \\
\text { Verputz }\end{array}$ & $\begin{array}{c}\text { Bois mort } \\
\text { Totes Holz }\end{array}$ \\
\hline $\begin{array}{l}\text { Nombre de nichoirs proposés } \ldots \ldots \ldots \ldots \\
\text { Zahl d. angebotenen Nistgelegenheiten. }\end{array}$ & 7 & 24 & 8 & 11 \\
\hline $\begin{array}{l}\text { Nombre de nids par nichoir. } \\
\text { Zahl d. Nester je Anlage. }\end{array}$ & & & & \\
\hline-0. cornuta.................. & 0 & 0,33 & 0,38 & 1,18 \\
\hline$-0 . r u f a \ldots \ldots \ldots \ldots \ldots \ldots \ldots \ldots$ & 0 & 0,79 & 0,25 & 1,09 \\
\hline
\end{tabular}


Tableav 7. - Répartition des nids suivant l'orientation des nichoirs. Lusignan 1971

TABELLE 7. - Verteilung der Nester nach der Himmelsrichtung der Nistanlagen. Lusignan 1971

\begin{tabular}{|c|c|c|c|c|}
\hline $\begin{array}{c}\text { Orientation } \\
\text { Himmelsrichtung }\end{array}$ & $\begin{array}{c}\text { Est } \\
\text { Osten }\end{array}$ & $\begin{array}{l}\text { Sud } \\
\text { Süden }\end{array}$ & $\begin{array}{l}\text { Ouest } \\
\text { Westen }\end{array}$ & $\begin{array}{l}\text { Nord } \\
\text { Norden }\end{array}$ \\
\hline $\begin{array}{l}\text { Nombre de nichoirs proposés } \ldots \ldots \ldots \ldots \\
\text { Zahl d. angebotenen Nistgelegenheiten. }\end{array}$ & 13 & 15 & 13 & 14 \\
\hline $\begin{array}{l}\text { Nombre de nichoirs occupés } \\
\text { Zahl der angenommenen Nistanlagen }\end{array}$ & & & & \\
\hline 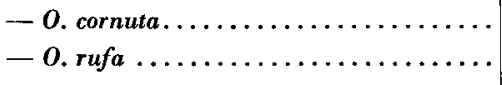 & $\begin{array}{l}1 \\
3\end{array}$ & $\begin{array}{l}0 \\
0\end{array}$ & $\begin{array}{l}4 \\
3\end{array}$ & $\begin{array}{l}7 \\
4\end{array}$ \\
\hline $\begin{array}{l}\text { Nombre total de nids } \\
\text { Gesamtzahl der Nester }\end{array}$ & & & & \\
\hline 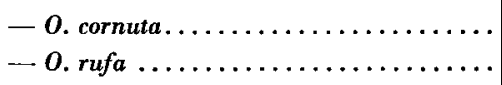 & $\begin{array}{l}2 \\
4\end{array}$ & $\begin{array}{l}0 \\
0\end{array}$ & $\begin{array}{r}9 \\
15\end{array}$ & $\begin{array}{l}13 \\
18\end{array}$ \\
\hline
\end{tabular}

TABLEaU 8. - Répartition des nids suivant le diamètre du tube. Lusignan 1971

TABELLE 8. - Verteilung der Nester nach dem Röhrchen-Durchmesser. Lusignan 1971

\begin{tabular}{|c|c|c|c|}
\hline $\begin{array}{l}\text { Diamètre en } \mathbf{m m} \\
\text { Durchmesser in } \mathbf{m m}\end{array}$ & 4 & 5 & 8 \\
\hline $\begin{array}{l}\text { Nombre de nids construits par } \\
\text { Anzahl der gebauten Nester von. }\end{array}$ & & & \\
\hline$\ldots \ldots \ldots \ldots \ldots \ldots \ldots \ldots$ & 6 & 9 & 9 \\
\hline$\ldots \ldots \ldots \ldots \ldots$ & 2 & 18 & 12 \\
\hline
\end{tabular}

Deux orientations sont préférées par les deux espèces : l'Ouest et le Nord. Les nichoirs exposés à l'Est sont beaucoup moins visités. Le Sud est la seule orientation totalement délaissée. Nos résultats sont en contradiction avec les suppositions d'un grand nombre d'auteurs qui estiment a priori que les orientations Sud et Est sont les plus favorables aux nidifications des Osmies. La tendance à préférer les expositions à l'Ouest et au Nord caractérise $O$. cornuta et $O$. rufa, tandis qu'une autre Osmie nidifiant dans nos ( piège-nichoir » en très grand nombre de mai à septembre Osmia coerulescens L. préfère les expositions Est et Sud (Tasei, 1972). Nous devons préciser que l'orientation des nichoirs à l'Ouest, expose l'entrée des nichoirs au vent dominant et à la pluie et provoque le développement de moisissures. 
Les trous de 5 et $8 \mathrm{~mm}$ de diamètre contiennent la grande majorité des nidifications chez les deux espèces. Les tubes de $4 \mathrm{~mm}$ ne servent que rarement, aux individus de taille inférieure à la normale. Nous avons eu l'occasion d'observer un nid établi par $O$. rufa dans un trou de $15 \mathrm{~mm}$ alors que des diamètres inférieurs étaient disponibles au même endroit. Divers auteurs donnent des diamètres du même ordre de grandeur que ceux qui figurent dans nos résultats.

Notre dispositif d'observation ne teste pas l'influence de l'inclinaison des tubes, puisque toutes les cavités proposées sont horizontales; aussi il est intéressant de signaler que d'après Hardouin (1948) et Bonelli (1966) o. rufa nidifie parfois dans des tubes ou roseaux verticaux alors que d'après le premier auteur l'horizontalité des tubes est une condition sine qua non de la nidification chez $O$. cornuta.

\section{5. - Activité de butinage et approvisionnement du nid}

a) Observations en serre sur le butinage

Les femelles prélèvent le nectar dans certaines fleurs, le pollen dans d'autres (quelques espèces sont visitées pour les deux substances). Enfin beaucoup d'espèces ne sont butinées ni pour le nectar, ni pour le pollen. Sur les fleurs en châtons comme le noisetier, le saule, etc. les femelles récoltent le pollen en grattant les fleurs avec les pattes I et II. Ce pollen s'accumule dans la brosse abdominale. Sur les fleurs hermaphrodites à corolle étalée comme les ficaires c'est la partie centrale du corps de la femelle qui frotte les étamines avec une grande rapidité. Les fleurs à corolle papilonacée sont ouvertes grâce à l'introduction du proboscis, puis les étamines sorties de la « carène » sont grattées avec les pattes antérieures.

Nous avons tenté d'estimer de façon rapide quelles sont les plantes à nectar, les plantes à pollen et les plantes non visitées, chez les deux espèces étudiées. Nous avons offert à $O$. rufa 32 espèces appartenant à 16 familles botaniques et à $O$. cornuta 29 espèces appartenant à 13 familles botaniques. Les croix des tableaux 9 et 10 indiquent les fréquences approximatives des visites pour le nectar ou pour le pollen.

$O$. rufa récolte le pollen surtout sur Renonculacées, Papaveracées, Rosacées, Crucifères, Bétulacées, Salicacées, Papilionacées. Certaines familles sont laissées à l'écart : Composées, Scrofulariacées, Primulacées, Liliacées, Caryophyllacées, Labiées, Borraginacées.

o. cornuta a un domaine de butinage pour le pollen plus restreint : Rosacées, Crucifères, Salicacées, Papilionacées. Les familles qui ne sont jamais butinées pour le pollen sont les mêmes que chez $O$. rufa (mise à part la famille des Composées).

Chez les deux espèces la famille des Rosacées est la seule dont toutes les espèces communes en avril-mai soient butinées pour le pollen. 
Tableau 9. - Fleurs nectarifères et pollinifères, O. rufa

TABelle 9. - Nektar- u. pollenspendende Blüten, O. rufa

\begin{tabular}{|c|c|c|c|}
\hline $\begin{array}{c}\text { Familles } \\
\text { Familien }\end{array}$ & $\begin{array}{c}\text { Espèces } \\
\text { Arten }\end{array}$ & $\begin{array}{c}\text { Nectar } \\
\text { Nektar }\end{array}$ & $\begin{array}{c}\text { Pollen } \\
\text { Pollen }\end{array}$ \\
\hline $\begin{array}{l}\text { Ranunculaceae } \\
\text { Papaveraceae } \\
\text { Gruciferae } \\
- \\
\text { Rosaceae } \\
- \\
- \\
\text { Plantaginaceae } \\
\text { Borraginaceae } \\
\text { Labiatae } \\
\quad- \\
\text { - } \\
\text { Papilionaceae } \\
\quad- \\
\quad- \\
\quad- \\
\text { - } \\
\text { Salicaceae } \\
\text { Liliaceae } \\
\text { Betulaceae } \\
\text { Compositae } \\
\text { Caryophyllaceae } \\
\text { Scrofulariaceae } \\
\text { Oleaceae } \\
\text { Primulaceae }\end{array}$ & $\begin{array}{l}\text { Ranunculus bulbosus } \\
\text { acris } \\
\text { *Ficaria ranunculoìdes } \\
\text { Caltha palustris } \\
\text { Anemone nemorosa } \\
\text { Papaver sp. } \\
\text { Brassica napus oleifera } \\
\text { Sinapis sp. } \\
\text { Prunus spinosa } \\
\text { Prunus sp. } \\
\text { Crataegus sp. } \\
\text { Pirus malus } \\
\text { Plantago lanceolata } \\
\text { Borrago officinalis } \\
\text { Pulmonaria sp. } \\
\text { *Lamium purpureum } \\
\text { Glechoma hederacea } \\
\text { Ajuga reptans } \\
\text { Vicia sepium } \\
\text { Trifolium repens } \\
\text { pratense } \\
\text { Onobrychis sativa } \\
\text { Medicago sativa } \\
\text { Ulex europeus } \\
{ }^{*} \text { Salix sp. } \\
\text { Endymion nutans } \\
\text { *Corylus avellana } \\
\text { Taraxacum sp. } \\
\text { Stellaria holostea } \\
\text { Veronica persica } \\
\text { *Forsythia } \\
\text { Primula officinalis }\end{array}$ & 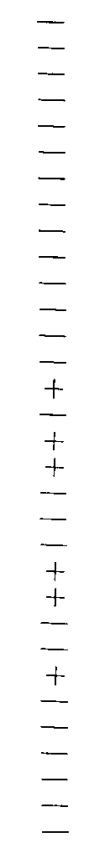 & $\begin{array}{c}+++ \\
+++ \\
++ \\
- \\
-\overline{+} \\
+++ \\
++ \\
++ \\
++ \\
++ \\
+++ \\
+ \\
- \\
- \\
- \\
- \\
++ \\
+ \\
- \\
+ \\
+++ \\
+++ \\
+ \\
- \\
- \\
-\end{array}$ \\
\hline
\end{tabular}

* Plantes dont la floraison ne chevauche pas les courbes d'émergence d'O. rufa dans les conditions naturelles.

* Pflanzen, deren Blütezeit unter natürlichen Bedingungen das Auftreten von $O$. rufa nicht überschneidet.

Sont butinées pour le nectar les Borraginacées, Labiées, Liliacées. Afin de mener à bien un élevage d' $O$. cornuta et $O$. rufa on a intérêt à présenter aux femelles des ( plantes à nectar ) et des ( plantes à pollen ).

Pour O. rufa : Ranunculus, Ficaria, Papaver, Trifolium, Brassica napus, Prunus, Pirus et Pulmonaria, Ajuga, Endymion... (Le saule et le noisetier fleurissent trop précocement et sont utilisés seulement lorsque les $O$. rufa sont réactivées en laboratoire.)

Pour O. cornuta : Brassica napus, Prunus, Pirus, Salix, Trifolium Pulmonaria, Medicago sativa, Lupinus, Endymion...

Nous avons eu l'occasion à plusieurs reprises de suivre des femelles au cours d'une série de voyages de butinage. Nous avons évalué lors de chaque “ test de préférence » et pour chaque espèce de plante, 3 caractéristiques : 
TABLeaU 10. - Fleurs nectarifères et pollinifères, 0 . cornuta

TABELLE 10. - Nehtar- und pollenspendende Blüten, O. cornuta

\begin{tabular}{|c|c|c|c|}
\hline $\begin{array}{c}\text { Familles } \\
\text { Familien }\end{array}$ & $\begin{array}{c}\text { Espèces } \\
\text { Arten }\end{array}$ & $\begin{array}{l}\text { Nectar } \\
\text { Nektar }\end{array}$ & $\begin{array}{c}\text { Pollen } \\
\text { Pollen }\end{array}$ \\
\hline $\begin{array}{l}\text { Ranunculaceae } \\
\text { Cruciferae } \\
\text { Rosaceae } \\
- \\
- \\
\text { Borraginaceae } \\
\text { Labiatae } \\
\text { Papilionaceae } \\
\quad- \\
\quad- \\
\quad- \\
\quad- \\
\text { Salicaceae } \\
\text { Liliaceae } \\
\text { Compositae } \\
\text { Caryophyllaceae } \\
\text { Scrofulariaceae } \\
\text { Oleaceae } \\
\text { Primulaceae }\end{array}$ & $\begin{array}{l}\text { Ranunculus bulbosus } \\
\text { Ficaria ranunculoïdes } \\
\text { Brassica napus oleifera } \\
\text { Capsella bursapastocea } \\
\text { Sisymbrium aliara } \\
\text { Cardamine sp. } \\
\text { Prumus spinosa } \\
\text { Pirus malus } \\
\text { Pirus communis } \\
\text { Prunus cerasus } \\
\text { Pulmonaria sp. } \\
\text { Borrago officinalis } \\
\text { Glechoma hederacea } \\
\text { Ajuga reptans } \\
\text { Melilotus alba } \\
\text { Lupinus sp. } \\
\text { *Medicago sativa } \\
\text { Trifolium pratense } \\
\text { Ulex europeus } \\
\text { Vicia sepium } \\
\text { Trifolium repens } \\
\text { Salix sp. } \\
\text { Endymion nutans } \\
\text { Taraxacum } \\
\text { Stellaria holostea } \\
\text { Veronica persica } \\
\text { Syringa } \\
\text { Primula officinalis }\end{array}$ & $\begin{array}{l}- \\
+ \\
- \\
+ \\
= \\
- \\
- \\
+ \\
++ \\
+ \\
+ \\
- \\
++ \\
++ \\
- \\
- \\
- \\
- \\
+ \\
+ \\
+ \\
-\end{array}$ & $\begin{array}{l}+ \\
+ \\
++ \\
+ \\
+ \\
+++ \\
++ \\
++ \\
+++ \\
- \\
- \\
- \\
+ \\
+ \\
= \\
+ \\
++ \\
+ \\
- \\
-\end{array}$ \\
\hline
\end{tabular}

* Plantes dont la floraison ne chevauche pas les courbes d'émergence d'O. cornuta dans les conditions naturelles.

* Pflanzen, deren Blütezeit unter natürlichen Bedingungen das Auftreten von $O$. cornula njcht überschneidet.

a) la durée du butinage (en \% du temps total);

b) la fréquence des visites (en \% du nombre total de visites);

c) le temps moyen d'une visite (en secondes).

Les tableaux 11, 12 et 13 présentent quatre analyses du comportement de butinage en serre, destinées à mettre en évidence les différences d'attractivité des fleurs pour 0 , cornuta et 0 . rufa.

Ces quelques tests de préférence nous apprennent que deux caractéristiques a) et b), varient généralement dans le même sens quand on passe d'une espèce botanique à une autre. Cependant cette règle se trouve en défaut lorsqu'une espèce très recherchée par l'abeille ne dispose plus que de fleurs fanées. Dans ce cas la fréquence des visites est élevée mais le temps de butinage est faible. En conséquence, lorsque l'on effectue un test de préférence en serre avec des fleurs fraîches il suffit de calculer la seule caractéristique a) = durée de butinage en pourcentage du temps total. 
Tableau 11. - Analyse de 18 minutes de butinage d'O. cornuta en serre

Tabelle 11. - Analyse von 18 Sammelminuten von O. cornuta im Gewächshaus

\begin{tabular}{|c|c|c|c|c|}
\hline $\begin{array}{c}\text { Espèce } \\
\text { Art }\end{array}$ & Salix & Prunus & Lupinus & Sisymbrium \\
\hline a) & 9,6 & 81,0 & 9,0 & 0,4 \\
\hline b) & 22,0 & 56,0 & 16,0 & 6,0 \\
\hline c) & 26 & 88 & 33 & 5 \\
\hline
\end{tabular}

a) durée de butinage (en \% du temps total).

b) fréquence des visites (en $\%$ du nombre total des visites).

c) temps moyen d'une visite (en secondes).

a) Dauer d. Sammeltätigkeit (in \% der Gesamtzeit).

b) Häufigkeit d. Besuche (in \% der Gesamtzahl).

c) Durchschnittliche Dauer eines Besuches (in Sek).

Tableau 12. - Analyse de 1 h $47 \mathrm{mn} 37 \mathrm{~s}$ de butinage d'O. cornuta en serre

TABELle 12. - Analyse einer Sammeltätigkeit von 1 Stde 47 Min 37 Sek von 0. cornuta im Gewächshaus

\begin{tabular}{|c|c|c|c|c|c|c|c|c|}
\hline $\begin{array}{c}\text { Espèce } \\
\text { Art }\end{array}$ & $\begin{array}{l}\text { Pulmo- } \\
\text { naria }\end{array}$ & $\begin{array}{c}\text { Endymion } \\
\text { nutans }\end{array}$ & $\begin{array}{l}\text { Pirus } \\
\text { commu- } \\
\text { nis }\end{array}$ & $\begin{array}{l}\text { Pirus } \\
\text { malus }\end{array}$ & $\begin{array}{l}\text { Prunus } \\
\text { cerasus }\end{array}$ & $\begin{array}{l}\text { Stellaria } \\
\text { holostea }\end{array}$ & $\begin{array}{c}\text { Brassica } \\
\text { napus } \\
\text { oleracea }\end{array}$ & $\begin{array}{l}\text { Tara- } \\
\text { xacum }\end{array}$ \\
\hline a) & 3,1 & 1,1 & 5,3 & 69,0 & 4,3 & 0,4 & 16,2 & 0,6 \\
\hline b) & 4,7 & 4,7 & 11,3 & 45,3 & 13,2 & 1,8 & 17,0 & 1,9 \\
\hline c) & 40 & 13 & 28 & 94 & 19 & 13 & 58 & 20 \\
\hline
\end{tabular}

$a), b), c$ ) comme pour le tableau 11 .

a) b) c) wie vorher.

Par ailleurs il ne semble pas que l'on ait à craindre les effets de surface dans des enceintes de dimensions réduites : une espèce peu attractive et abondante est toujours bien moins visitée qu'une fleur très attractive mais peu abondante.

Les tests prouvent la préférence d'O. cornuta pour : Prunus, Pirus malus, Brassica, Salix et la préférence d'O. rufa pour : Salix, Pirus malus, Brassica napus oleracea, Ranunculus et Papaver. Le tableau 13 compare les réactions de deux femelles d'O. rufa. Ces réactions sont identiques vis-à-vis de Pirus et Brassica, mais elles sont différentes vis-à-vis d'Endymion, Ranunculus, Trifolium, Salix, Ajuga et Plantago. Tous ces résultats confirment et précisent 
Tableau 13. - Analyse de 1 h $12 \mathrm{mn}$ de butinage d'O. rufa $n^{\circ} 1 \mathrm{~A}$ $2 \mathrm{~h} 16 \mathrm{mn}$ de butinage d'O. rufa $n^{\circ} 2 \mathrm{~B}$

TABELle 13. - Analyse einer Sammeltätigkeit von 1 Stde $12 \mathrm{Min}$ von $\mathrm{O}$. rufa $\mathrm{Nr}$. I A u.

2 Stden $16 \mathrm{Min}$ von $\mathrm{O}$. rufa $\mathrm{Nr} .2 \mathrm{~B}$

$\mathbf{A}$

\begin{tabular}{|c|c|c|c|c|c|c|c|c|c|}
\hline $\begin{array}{l}\text { Espèce } \\
\text { Art }\end{array}$ & $\begin{array}{l}\text { Pirus } \\
\text { malus }\end{array}$ & Endymion & Papaver & $\begin{array}{l}\text { Ranun- } \\
\text { culus }\end{array}$ & $\begin{array}{c}\text { Brassica } \\
\text { napus } \\
\text { oleracea }\end{array}$ & $\begin{array}{c}\text { Trifolium } \\
\text { repens }\end{array}$ & Salix & Ajuga & Plantago \\
\hline a) & 23 & 10 & 6 & 4 & 23 & 1 & 4 & 4 & - \\
\hline b) & 25 & 8 & 21 & 8 & 20 & 4 & 8 & 6 & - \\
\hline c) & 31 & 43 & 10 & 19 & 43 & 8 & 15 & 21 & - \\
\hline
\end{tabular}

$\mathbf{B}$

\begin{tabular}{|c|c|c|c|c|c|c|c|c|c|}
\hline a) & 25 & - & 0,5 & 44 & 22 & 0,5 & - & - & 0,5 \\
\hline b) & 32 & - & 4 & 32 & 28 & 2 & - & - & 2 \\
\hline c) & 35 & - & 5 & 62 & 36 & 22 & - & - & 13 \\
\hline
\end{tabular}

- a), b), c), comme pour les tableaux 11 et 12 ;

- les 2 femelles ont été observées le même jour sur le même ensemble de fleurs.

a) b) c) wie vorher.

Die beiden Weibchen wurden am gleichen Tag am gleichen Bluitenbestand beobachtet.

Tableau 14. - Durées de voyage de butinage chez 0 . cornuta et 0 . rufa en serre

TABeLLe 14. - Dauer der Sammelflüge von O. cornuta und O. rufa im Gewächshaus

\begin{tabular}{|c|c|c|c|}
\hline & Nombre de voyages & $\begin{array}{l}\text { Durée moyenne } \\
\text { d'un voyage }\end{array}$ & $\begin{array}{l}\text { Temps extrêmes } \\
\text { des voyages }\end{array}$ \\
\hline Art & Zahl der Flüge & Mittlere Dauer eines Fluges & Extreme Flugdauer \\
\hline O. cornuta $\mathrm{n}^{\circ} 1 \ldots \ldots \ldots$ & 18 & $5 \mathrm{mn} 42 \mathrm{~s}$ & $1 \mathrm{mn}$ à $13 \mathrm{mn}$ \\
\hline O. cornuta $\mathrm{n}^{\circ} 2 \ldots$ & 12 & $4 \mathrm{mn} 42 \mathrm{~s}$ & $1 \mathrm{mn} 10 \mathrm{~s}$ à $8 \mathrm{mn} 19 \mathrm{~s}$ \\
\hline O. $r u f a n^{\circ} 1 \ldots \ldots$ & 21 & $8 \mathrm{mn} 42 \mathrm{~s}$ & $4 \mathrm{mn}$ à $17 \mathrm{mn}$ \\
\hline O. rufa no $2 \ldots$ & 15 & $6 \mathrm{mn} 06 \mathrm{~s}$ & $2 \mathrm{mn}$ à $12 \mathrm{mn}$ \\
\hline
\end{tabular}

les données des tableaux 9 et 10 . On doit souligner que les observations concernant le comportement de butinage doivent porter sur plusieurs femelles puisque 
l'on a mis en évidence des différences de comportement chez des femelles de la même espèce, placées strictement dans les mêmes conditions.

Les temps moyens du chargement de la brosse sont 5 à $6 \mathrm{mn}$. chez O. cornuta et 6 à $9 \mathrm{mn}$. chez $O$. rufa (tableau 14), mais les deux espèces effectuent tantôt des voyages de longue durée (8 à $17 \mathrm{mn}$.), tantôt des voyages de courte durée (1 à $4 \mathrm{mn}$.). Cette alternance a été observée chez Osmia lignaria : voyages longs de 22 à 45 minutes et voyages courts de 5 à 10 minutes (Levis, 1966). Bonelli, (1966) donne des temps de voyage de butinage de 12 à 19 minutes pour $O$. rufa. Il est probable que les conditions de la serre permettent à l'insecte un chargement plus rapide de sa brosse.

b) L'approvisionnement des cellules en serre.

$O$. cornuta approvisionne complètement une cellule en 4 heures $30 \mathrm{mn}$. 5 heures. Il lui faut donc effectuer 36 à 40 voyages de butinage.

o. rufa approvisionne une cellule dans les mêmes temps qu'O. cornuta. Il lui faut effectuer une trentaine de voyages de butinage par cellule.

Au cours d'une journée ensoleillée on peut estimer qu'une Osmie effectue 70 à 100 voyages de butinage (compte tenu des travaux de maçonnerie effectués pour fermer les cellules). De telles observations directes de l'approvisionnement des cellules sont relatées par Hartman (1944) chez Osmia georgica. Cette espèce effectue 35 à 40 voyages pour garnir une cellule. Malyshev (1935) pense qu'il suffit de compter les couches composant la provision de pollen, pour connaître le nombre de voyages effectués par une femelle; c'est ainsi qu'il déduit qu'O. rufa fait 5 à 6 voyages par cellule. Sa méthode est donc peu précise.

Les provisions d' $O$. cornuta pèsent en moyenne $0,39 \mathrm{~g}$. Les poids des provisions des 9 cellules ayant servi aux mesures varient de $0,36 \mathrm{~g}$ à $0,43 \mathrm{~g}$ (poids frais). La femelle transporte done en moyenne $0,011 \mathrm{~g}$ de pollen et de produits liquides régurgités à chacun des voyages.

Les provisions d'O. rufa pèsent en moyenne $0,29 \mathrm{~g}$. Les poids des provisions de 32 cellules varient de 0,17 à $0,37 \mathrm{~g}$. La femelle transporte en moyenne $0,010 \mathrm{~g}$ de pollen et de nectar à chacun de ses voyages. Ces données sont celles des cellules construites en serre. Les provisions de 26 cellules construites dans la nature par 0 . rufa pèsent en moyenne $0,26 \mathrm{~g}$ (de 0,15 à $0,41 \mathrm{~g}$.). Il ne semble pas que les conditions de serre modifient l'approvisionnement.

La teneur en eau des provisions varie de 18,5 à $20 \%$ chez $O$. cornuta et de 13,5 à $36,9 \%$ chez $O$. rufa.

Comme d'autres auteurs l'ont noté nous avons remarqué que dans certains nids les grosses provisions de pollen sont placées dans les cellules à femelle et les petites dans les cellules à mâle. Cependant il ne s'agit pas d'une règle 
immuable et souvent on ne peut pas prévoir le sexe de l'individu par le simple examen de sa provision de nourriture.

La femelle qui vient de garnir sa brosse de pollen entre dans son nid la tête la première, régurgite du nectar et tasse le « pain d'abeille » avec son front. Heroin-Delaunay, (1966), a démontré que des sécrétions imprègnent les provisions d'O. cornuta. Puis la femelle sort du nid, se retourne, entre à reculons et se décharge du pollen en grattant sa brosse avec ses pattes III. Lorsque le diamètre du tube est assez fort la femelle opère le retournement de son corps à l'intérieur du tube. La séquence de l'approvisionnement est identique chez les deux espèces : apport de nectar-malaxage-apport de pollen. Cependant l'aspect général des provisions est différent chez $O$. cornuta et $O$. rufa. Les provisions d'O. rufa sont recouvertes de pollen en poudre alors que celles d'O. cornuta sont des amas homogènes aussi humides en surface qu'à l'intérieur.

Une femelle d' $O$. cornuta élevée en serre passait en moyenne 1 minute 45 secondes à l'intérieur du nid. 56 secondes étaient consacrées à l'apport de nectar et 48 secondes au déchargement du pollen (Temps extrêmes : 35 secondes et 60 secondes). Deux femelles d'O. rufa passaient respectivement 1 minute 16 secondes et 1 minute dans leur nid. Les temps de régurgitation du nectar étaient de 10 et 23 secondes (Temps extrêmes : 10 secondes et 35 secondes); les temps de déchargement du pollen étaient de 56 et 37 secondes (Temps extrêmes : 20 secondes et 120 secondes). Si l'on se fie à ces trois exemples, il semble qu'O. cornuta soit plus lente (ou plus a soigneuse ") qu'O. rufa quand elle régurgite le nectar et malaxe la provision.

c) Le butinage dans les conditions naturelles de Lusignan.

L'analyse microscopique des provisions de 31 cellules d'O. cornuta et de 52 cellules d' $O$. rufa construites dans les conditions naturelles nous permet d'établir une liste des plantes butinées par les deux espèces (tableau 15).

Tableau 15. - Plantes butinées pour le pollen par 0. cornuta et 0 . rufa dans les conditions naturelles. Lusignan 1971

Tabelle 15. - Pfanzen, die unter natïrlichen Bedingungen von O. cornuta und 0 . rufa des Pollens wegen beflogen wurden. Lusignan 1971

\begin{tabular}{|c|c|}
\hline O. cornuta & O. rufa \\
\hline $\begin{array}{l}\text { - Prunus sp. (P. cerasus L. la plupart du temps). } \\
\text { - Pirus communis L. } \\
\text { - Pirus malus L. } \\
\text { - Crataegus L. } \\
\text { - Salix L. } \\
\text { - Tulipa L. } \\
\text { - Taraxacum Juss. } \\
\text { - Quercus pedunculata Ehrh. } \\
\text { - Ranunculus bulbosus L. } \\
\text { - Brassica Napus L. (var. oleifera D.C.) } \\
\text { - Ilex aquifolium L. }\end{array}$ & $\begin{array}{l}\text { - Prunus sp. } \\
\text { - Pirus communis } \\
\text { - Pirus malus } \\
\text { - Crataegus L. } \\
\text { - Rosa sp. } \\
\text { - Quercus pedunculata } \\
\text { - Ranunculus bulbosus L. et } \text { R. acris L. } \\
\text { - Brassica Napus oleifera } \\
\text { - Ilex aquifolium } \\
\text { - Papaver sp. } \\
\text { - Helianthemum sp. } \\
\text { - Stellaria Holostea } L . \\
\text { - Vicia L. }\end{array}$ \\
\hline
\end{tabular}


$O$. cornuta récolte le pollen sur 11 genres appartenant à 8 familles botaniques : Rosacées, Salicacées, Liliacées, Composées, Fagacées, Renonculacées, Crucifères, Ilicinées.

$O$. rufa récolte le pollen sur 12 genres appartenant à 9 familles botaniques : Rosacées, Fagacées, Renonculacées, Crucifères, Ilicinées, Papaveracées, Cistacées, Caryophyllacées, Papilionacées.

Ces analyses confirment les résultats de l'observation du butinage en serre. On peut dire que $O$. cornuta et $O$. rufa sont polylectiques. On trouvera les définitions et l'utilisation des termes ( polylectiques », (c oligolectiques ) et “ monolectiques » dans Robertson (1925), Linsley (1958) et Tasei (1972).

On peut rencontrer des provisions composées de plusieurs espèces de pollens aussi bien que des provisions « uniflorales ». Le tableau 16 indique que les provisions uniflorales représentent la moitié des provisions chez les deux espèces. Les provisions composées de deux espèces de pollens représentent

Tableau 16. - Association de pollens dans une même cellule chez 0 . cornuta et 0 . rufa dans les conditions naturelles. Lusignan 1971

TABelde 16. - Vorkommen verschiedener Pollenarten in einer Zelle bei O. cornuta und O. rufa unter natïrlichen Bedingungen. Lusignan 1971

\begin{tabular}{|c|c|c|c|c|c|}
\hline \multicolumn{2}{|c|}{$\begin{array}{c}\text { Nombre d'espèces de pollens par cellule } \\
\text { Zabl der Pollenarten je Zelle }\end{array}$} & 1 & 2 & 3 & 4 \\
\hline \multirow{2}{*}{$\begin{array}{l}\text { Nombre de cellules } \\
\text { Zahl d. Zellen }\end{array}$} & O. cornuta......... & 18 & 10 & 2 & 1 \\
\hline & o. rufa $\ldots \ldots \ldots \ldots$ & 25 & 16 & 7 & 4 \\
\hline
\end{tabular}

le tiers des effectifs. Les autres provisions sont composées de 3 ou 4 espèces de pollens.

Les provisions uniflorales sont constituées chez $O$. cornuta par les pollens de : Prunus sp., Pirus malus, Ranunculus; chez O. rufa par les pollens de : Quercus, Ranunculus, Papaver. Les provisions composites sont formées chez O. cornuta par les associations suivantes :

Prunus + Taraxacum; Prunus + Quercus; Crataegus + Ranunculus; Crataegus + Quercus + Ranunculus + Brassica.

Chez O. rufa: Pirus communis + Quercus; Crataegus + Quercus; Pirus malus + Quercus + Ranunculus; Ranunculus + Papaver; Ranunculus + Vicia; Rosa + Ranunculus + Papaver + Helianthemum.

Dans certains nids nous avons analysé le contenu de la première et de la dernière cellule. Trois nids sur sept chez $O$. cornuta et trois nids sur seize chez 
O. rufa étaient approvisionnés dans ces deux cellules avec des pollens différents. Ceci prouve qu'une femelle peut modifier en 4 ou 5 jours son comportement vis-à-vis des plantes qui l'environnent.

La figure 3 représente les volumes des divers pollens récoltés par $O$. cornuta et $O$. rufa pendant la période de vol. Chez $O$. cornuta le pollen de Prunus est de loin le plus abondamment récolté, tous les autres sont considérés comme des pollens d'accompagnement. Chez $O$. rufa le pollen de Quercus est le pollen principal, celui de Ranunculus qui représente un volume respectable, est le pollen secondaire; les autres sont des pollens d'accompagnement (rosacées surtout). Ces deux Osmies sont bien polylectiques mais manifestent une tendance à l'oligolectie c'est-à-dire une tendance à ne butiner qu'une espèce ou une famille de plantes. Cette tendance serait plus marquée chez $\boldsymbol{O}$. cornuta.

Le tableau 17 donne les spectres polliniques des espèces récoltées dans les 6 localités possédant des nichoirs. Il n'est pas possible de comparer entre eux ces spectres car certains correspondent à l'analyse d'un trop petit nombre de cellules. Cependant nous pouvons dire que chez O. cornuta, Prunus est toujours le pollen principal. Dans une localité, Ranunculus est le pollen secondaire ( + de $25 \%$ ). Chez 0 . rufa le pollen principal est Quercus ou Ranunculus suivant la localité. Il est donc probable que la composition des flores locales ait une influence sur le spectre de récolte pollinique et que la raréfaction de la plante habituellement recherchée puisse inciter les Osmies à récolter en grande quantité un pollen ordinairement secondaire ou d'accompagnement.

Le tableau 18 représente les périodes de floraison des plantes butinées et les spectres polliniques hebdomadaires. On remarque de suite que certaines espèces sont butinées pendant toute leur floraison : Prunus, Pirus, Crataegus, d'autres ne sont visitées que lorsque la floraison atteint sa plus forte densité : Ranunculus, Papaver, enfin quelques plantes sont butinées accidentellement : Helianthemum, Ilex, Tulipa, Vicia sativa, Stellaria Holostea.

Lorsque les floraisons se succèdent les abeilles passent d'une espèce à l'autre sans attendre la fin des espèces les plus précoces; c'est ainsi qu'O. cornuta butine début avril sur Prunus, fin avril sur Pirus communis et Pirus malus, début mai sur Pirus malus et Crataegus fin mai sur Crataegus. O. rufa butine début mai sur Quercus, puis sur Quercus et Ranunculus, fin mai sur Ranunculus, et début juin sur Ranunculus et Papaver.

Les auteurs donnent en général des indications très brèves sur le butinage d'O. cornuta et $O$. rufa : O. cornuta est signalée sur amandier (FABRE 1913); sur Mahonia, pulmonaire, lilas, Veronica filiformis (HARdouin, 1948); sur Salix, Helleborus, abricotier, poirier, pommier, cerisier, prunier (IUGA, 1962).

$O$. rufa est signalée sur saule, hellébore, abricotier, poirier, pommier, cerisier (IugA, 1962).

Ces auteurs ne précisent généralement pas si les abeilles récoltent du nec- 


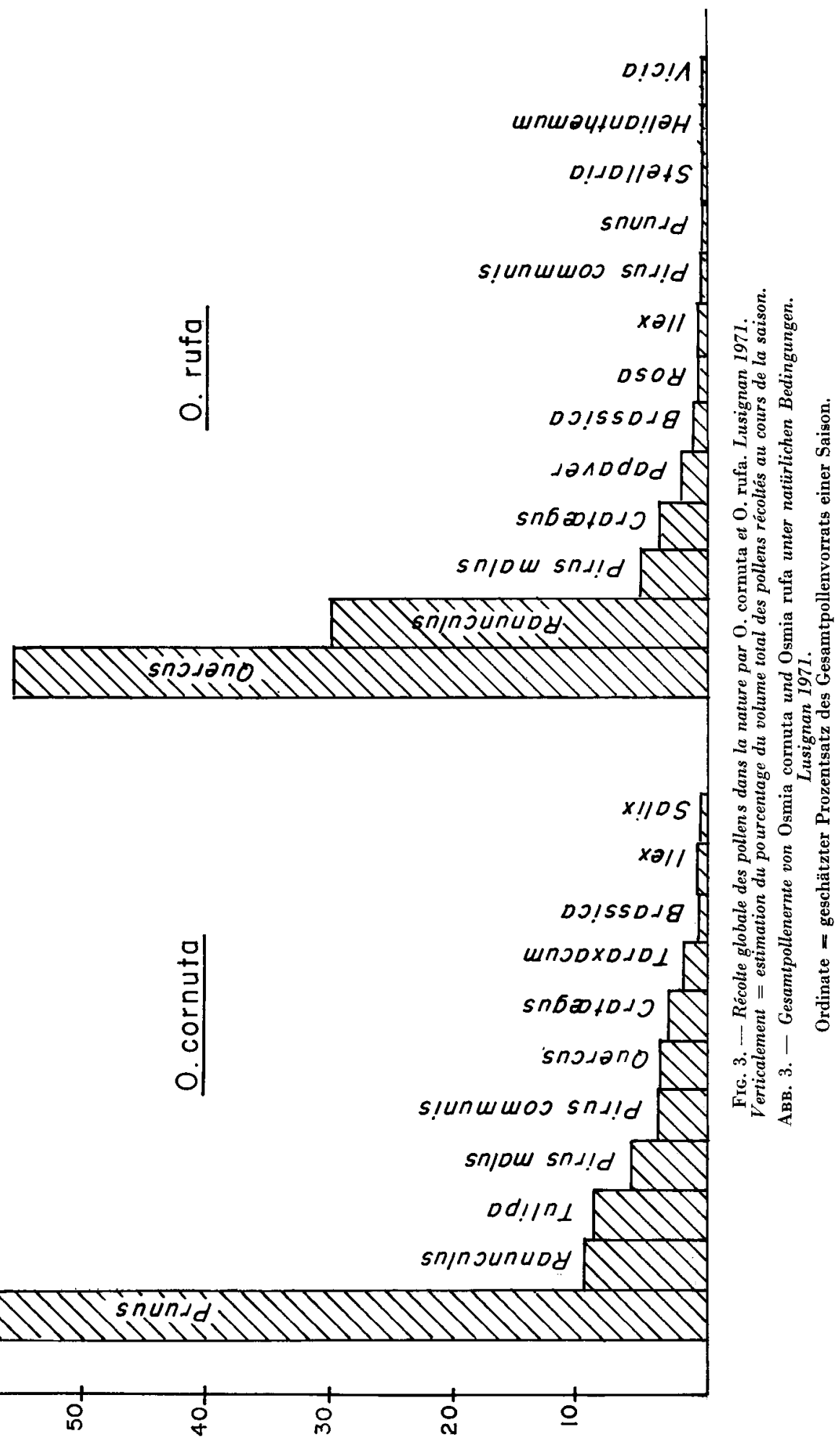




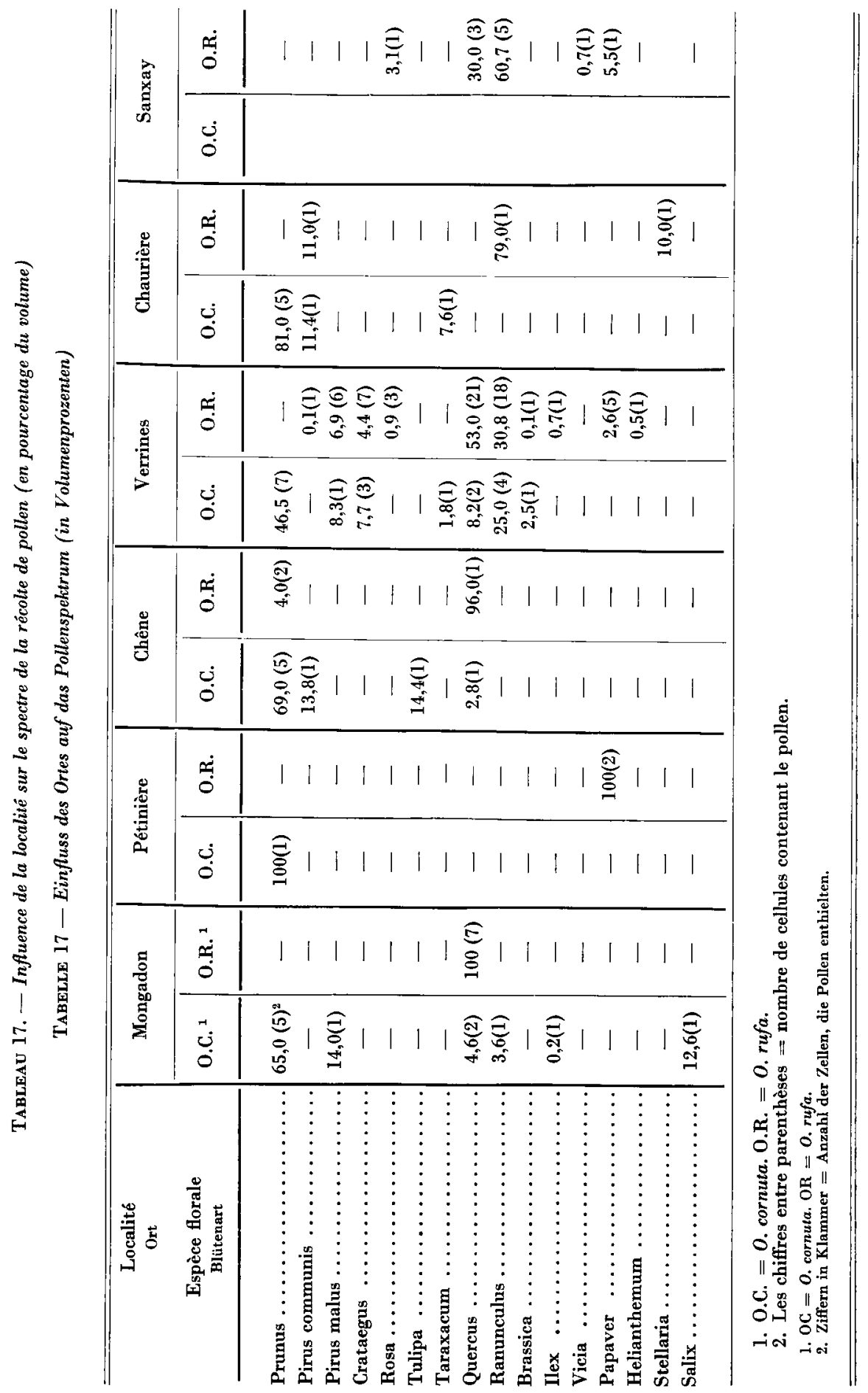




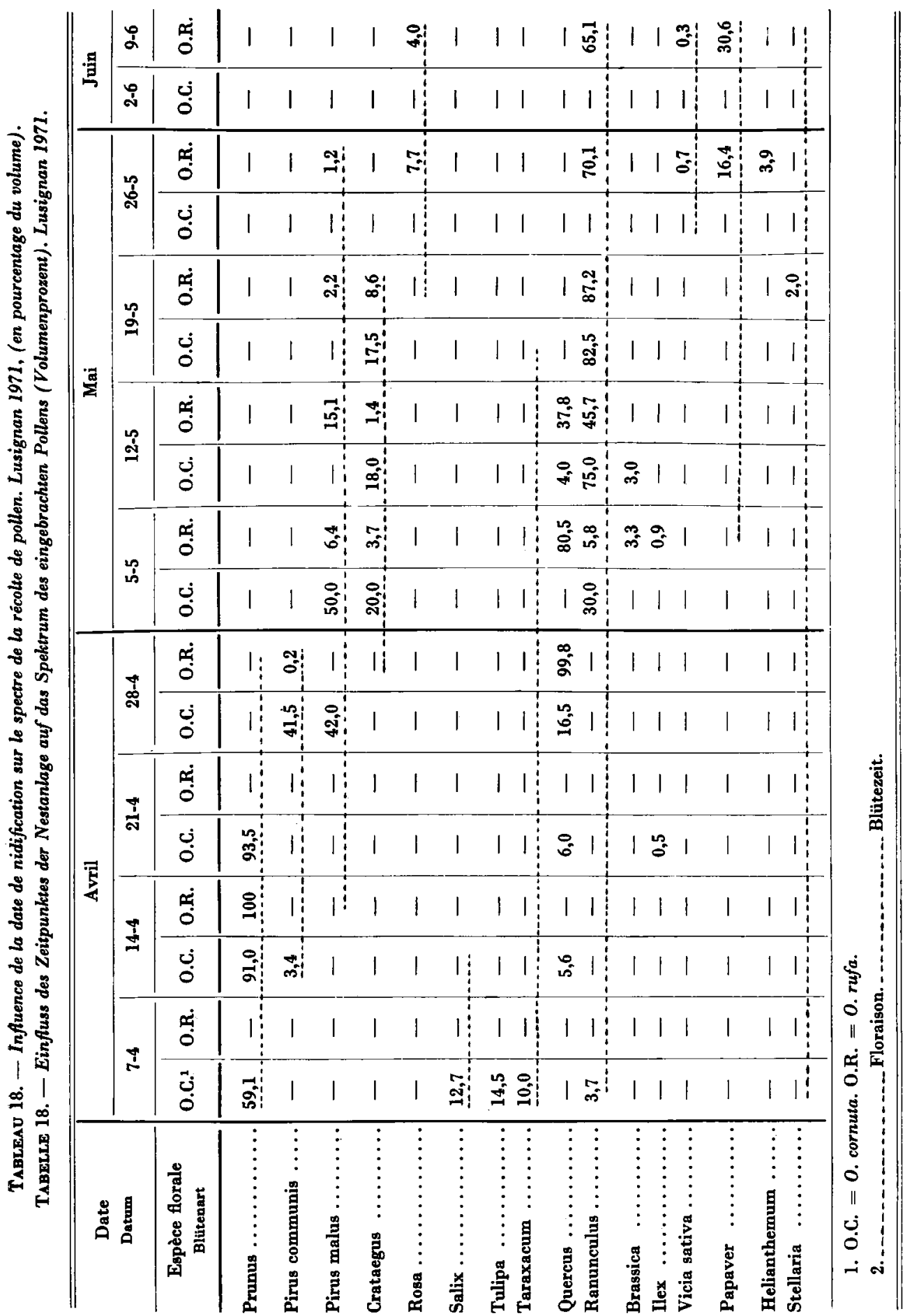


tar ou du pollen ou les deux substances, sur les plantes indiquées. LEcomte (1965, non publié) a réalisé quelques préparations microscopiques de provisions polliniques d'O. cornuta nidifiant dans la région parisienne. Les pollens récoltés sont : Prunus, Pirus, Acer, Ranunculus, Endymion, Salix, Quercus. D'après cet auteur, les populations d'O. cornuta peuvent être très favorisées par la floraison des saules, lorsque celle-ci a lieu pendant la période de nidification de l'Osmie. D'autres renseignements proviennent de Free et Williams (1970) qui ont analysé les provisions des cellules d'O. rufa dans diverses localités d'Angleterre. D'après ces auteurs $O$. rufa récolte surtout du pollen de Quercus robur, Ranunculus et Rubus (dans l'ordre l'importance décroissante). Ces résultats sont très proches des nôtres (Quercus pedunculata, Ranunculus, Rosacées). De plus Free et Williams remarquent que suivant la localité un pollen principal d'ordinaire peut devenir pollen mineur et vice-versa.

Nous considérons qu' $O$. cornuta est polylectique avec une tendance à butiner les Rosacées. $O$. rufa est plus polylectique d'importantes récoltes s'effectuant sur Cupulifères et Renonculacées. Le comportement polylectique d' $O$. cornuta est à rapprocher de celui d'O. lignaria qui a tendance à butiner une seule plante : Hydrophyllum capitatum (Levin, 1966) tandis que celui d'O. rufa se rapproche plutôt de celui d'une colonie d'abeilles domestiques (LouveauX, 1959).

Les tendances à l'oligolectie des Osmies sont rapportées par de nombreux auteurs : Friese, 1923; Malysheva, 1958; Miyamoto, 1959; Ponomavera, 1967 ; Marikovskaya, 1968...

En fait, dans le genre Osmia on trouve toutes les gradations entre le type : $O$. rufa et le type spécialisé dans la récolte du pollen d'un seul genre botanique :

- $O$. pedicornis récolte le pollen sur 5 familles mais a tendance à récolter surtout sur Ranunculus. (MiYamoto, 1959).

- O. coerulescens récolte le pollen sur 2 familles (Papilionacées et Labiées), butine assez rarement les Labiées (TASEI, 1972).

- O. californica récolte le pollen sur 1 famille (Composées) mais butine surtout sur Balsamorrhiza (Levin, 1966).

- $O$. adunca est réputée pour son butinage exclusif sur Echium.

Les deux premières espèces sont polylectiques et manifestent une tendance à l'oligolectie, forte chez $O$. pedicornis, très forte chez $O$. coerulescens. $O$. californica est oligolectique et $O$. adunda est monolectique.

Le spectre de récolte de pollen témoigne de la coïncidence dans le temps de la pleine floraison de certaines plantes et de la période de nidification des abeilles. Ce spectre résulte de la combinaison de deux tendances : la tendance héréditaire à butiner quelques plantes plus que d'autres et la tendance à visiter les plantes les plus abondantes. 


\section{6. - Le comportement de ponte}

Nous avons observé le comportement de ponte d' $O$. rufa, une seule fois au travers d'un tube de verre. La femelle dépose un dernier chargement de pollen, se retourne et le tasse puis se retournant à nouveau, elle allonge son abdomen, sort son aiguillon et laisse apparaître les $2 / 3$ de la longueur de l'œuf pendant 1 minute. Elle applique l'extrémité de l'œuf sur la provision de pollen, puis le libère complètement et avant de sortir du nid gratte deux ou trois fois sa brosse avec ses pattes postérieures. L'œuf est ainsi légèrement saupoudré de pollen. Nous avons pu constater que tous les oufs d'O. rufa portent des traces de pollen alors que ceux d' $O$. cornuta sont indemnes. Cela nous laisse supposer que le saupoudrage est un comportement constant chez $O$. rufa et qu'il n'a jamais lieu chez $O$. cornuta.

\section{7. - Étude expérimentale du comportement de nidification}

Nous avons observé en serre les réactions de 3 femelles $O$. cornuta no 1 , O. rufa $\mathrm{n}^{\circ} \mathrm{I}, O$. rufa $\mathrm{n}^{\mathrm{0}} 2$, à diverses perturbations survenant au cours de leur nidification.

a) O. cornuta $\mathrm{n}^{0} 1$

A 15 h 05 la femelle approvisionne une cellule. Pendant un de ses voyages de butinage on dispose devant la provision une cloison de terre sèche, empruntée à un autre nid, et on ajoute à cet opercule des granules de terre humide a fin d'imiter une construction neuve. La femelle revient avec son chargement de pollen et voyant la cloison va inspecter les tubes vides voisins de son nid, revenant 4 fois à sa cellule. Ce trouble dure 45 secondes, au bout desquelles l'insecte enlève l'opercule et dépose nectar et pollen comme il fait d'habitude. Cependant au lieu de repartir butiner de suite, il reste plus de 10 minutes dans son nid.

A 17 h 05 la cellule étant toujours en cours d'approvisionnement, on complète la provision avec du pollen sec récolté l'année précédente par o. cornuta. La femelle revenant avec son chargement hésite pendant 2 minutes, s'approchant et s'éloignant par 5 fois de sa cellule, puis régurgite son nectar (2 mn) et dépose son pollen (135 s) sur l'apport artificiel. La femelle sort pour butiner $1 \mathrm{mn} 12 \mathrm{~s}$ et revient humecter le pollen. Cette cellule contiendra un œuf et sera fermée comme les autres.

b) O. rufa $\mathrm{n}^{\circ} 1$

A 15 h 05 comme chez 0 . cornuta $\mathrm{n}^{0} 1$ on ajoute une cloison et des granules de terre humide. La femelles revenant avec son chargement de pollen inspecte la cloison, sort du nid, se repose. Ce manège est répété 5 fois en $2 \mathrm{mn}$ puis 
l'Osmie, retire la cloison, dépose son nectar et son pollen et reprend son activité normale.

A 15 h 10 on complète la provision avec du pollen sec récolté l'année précédente par $O$. rufa. La femelle revenant avec son chargement ne manifeste aucune hésitation et évacue le pollen étranger par paquets plus ou moins gros, qu'elle lache en plein vol. Ce déblaiement dure $8 \mathrm{mn}$.

A 15 h 35 on enlève tout le pollen de la cellule. A son retour la femelle hésite $5 \mathrm{mn}$ avant de déposer son nectar et son pollen. En effet par 7 fois elle entre dans la cellule et ressort très rapidement pour voler près du nid, ou bien se reposer ou bien butiner.

A $16 \mathrm{~h} 10$ le pollen primitif est remis dans la cellule. La femelle hésite $3 \mathrm{mn}$ avant de reprendre son comportement normal.

A $17 \mathrm{~h} 10$ la femelle a pondu et a fait son premier apport de boue pour fermer la cellule. On enlève l'œuf. L'Osmie ne manifeste aucun trouble et poursuit sa maçonnerie. On enlève alors tout le pollen de la cellule. La femelle continue d'élever la cloison de la cellule absolument vide.

Le lendemain à $11 \mathrm{~h}$ le bouchon terminal du nid est en cours de construction. On termine le travail en appliquant à la boue un bouchon étranger et en le mouillant. La femelle hésite devant son bouchon : elle fait alterner des périodes d'exploration de la partie externe du nid et des périodes de repos de 1 à $2 \mathrm{mn}$. Après $15 \mathrm{mn}$ de ce manège elle commence à gratter le bouchon comme pour l'enlever. Enfin à 12 h 36 elle adopte la solution définitive : égalisation de la surface du bouchon par quelques apports de boue et polissage de la maçonnerie.

A 17 h la seule cavité de nidification disponible est un tube en verre dont une extrémité est arrondie. La première construction n'a lieu que le lendemain à 11 h 40. Exceptionnellement la femelle ne construit pas de bouchon initial, elle se contente d'élever un “ pas de porte ». Cette construction est fréquemment interrompue et le premier apport de pollen n'a lieu que le lendemain à 15 h 50 . A plusieurs reprises au cours de l'approvisionnement ou de la construction on sort le tube de verre au soleil. La femelle n'est absolument pas perturbée et continue à travailler dans sa cellule en pleine lumière.

On déplace de 1 mètre le bac de terre humide où l'Osmie a l'habitude de malaxer de la boue. La femelle en train de bâtir une cloison est désorientée et ne construit plus. Le comportement normal est rétabli lorsque l'on remet le bac à sa place primitive.

On déplace le bac d'une très courte distance (d'une longueur de bac). La femelle est à nouveau désorientée, survolant l'emplacement primitif. Elle interrompt la construction pendant $14 \mathrm{mn}$ d'hésitations, de butinage, de survol de bac. Enfin elle se pose sur le bac déplacé et reprend la construction en acceptant le deuxième emplacement du bac. 
c) O. rufa no 2

Au cours de l'approvisionnement on complète la provision comme précédemment. La femelle de retour au nid hésite pendant une douzaine de minutes, puis reprend son butinage, humecte le pollen sec étranger et pond. La provision de pollen dépasse le “ pas de porte », la femelle élève la cloison sans pouvoir tenir compte de ce repère et sa construction trop proche de l'œuf le crève. Cela ne trouble pas le comportement de l'Osmie.

On dépose une cloison sèche. Immédiatement elle est éliminée par l'insecte qui poursuit ensuite sa propre construction.

On supprime la cloison en cours de construction. La femelle élimine les débris et se remet à la maçonnerie.

La cloison étant à nouveau ébauchée, on supprime tout le pollen. Cela ne modifie en rien le comportement de la femelle.

Avant la fin de cette construction on ferme le nid avec un bouchon terminal. La femelle hésite $3 \mathrm{mn}$ avant d'éliminer en $1 \mathrm{mn} 50 \mathrm{~s}$ ce bouchon artificiel et de poursuivre la construction de la cloison.

On déplace d'une longueur de bac le bac à terre. Un vol de reconnaissance de 12 s suffit à la femelle pour le retrouver. Au voyage suivant, on remet le bac à sa place primitive. La femelle se dirige immédiatement vers lui.

Au cours de la construction du bouchon terminal, le tube est enlevé, sa place étant occupée par un tube bouché. Pendant $3 \mathrm{mn}$ la femelle examine tous les tubes du nichoir mais surtout à l'emplacement primitif. Son nid est échangé avec un tube vide. En $1 \mathrm{mn}$ il est retrouvé mais pendant plus d'une demi-heure, à chaque retour, la femelle esquisse un détour par l'emplacement primitif.

De cette analyse expérimentale plusieurs conclusions sont tirées :

Les Osmies éliminent les constructions artificielles lorsqu'elles empêchent de poursuivre normalement la séquence de nidification. Si l'observateur complète une construction en cours, ou un approvisionnement, la femelle peut refuser ces apports étrangers, mais elle peut également accepter la situation nouvelle en terminant le travail de l'observateur (polissage de la construction étrangère, apport de nectar sur le pollen étranger).

Si on détruit une cloison en cours de construction, la femelle reprend son travail.

Après la ponte, l'élimination de l'ouf et des provisions n'est aucunement prise en considération.

Descy (1968) relate les réactions de nombreux individus à ses tracasseries. Il constate une grande hétérogénéité de comportement entre les femelles. 
Cependant quelques règles générales se dégagent de l'ensemble des observations : il existe une séquence de nidification type : cloison, apport de nectar, apport de pollen, ponte en fin d'approvisionnement, cloison, chez $O$. cornuta; cloison, " pas de porte ", apport de nectar, apport de pollen, ponte en fin d'approvisionnement, operculation du ( pas de porte ) chez 0 . rufa. Chaque fraction de séquence doit être achevée avant que soit commencée la suivante. Dès qu'une fraction est commencée, l'Osmie ne revient pas à la précédente même si des réparations ou même une deuxième ponte sont nécessaires. L'emplacement du nid est repéré grâce à la vision. L'olfaction peut jouer un rôle à courte distance puisque l'Osmie peut faire la distinction entre les matériaux étrangers et ceux qu'elle a pétris avec sa salive (et probablement imprégnés de son odeur). Le repérage visuel semble établi une fois pour toutes, car un tube étant bouché et remplacé par un tube vide, c'est le tube vide que s'approprie la femelle. Si le premier nid terminé reste en place, c'est le tube le plus proche qui est emprunté.

Descy note des anomalies dans certaines séquences de nidifications : une $O$. cornuta remplace un ouf d'O. rufa par le sien. Une $O$. rufa édifie un bouchon initial et abandonne le nid. Une Osmie pond deux œufs sur la même provision. Une autre dépose dans sa cellule un chargement de pollen après avoir pondu. Hardouin (1948) remarque des abérrations, telles que la destruction de cellules par la bâtisseuse elle-même.

Descy explique les anomalies de séquence par un manque de maturation du plus gros des ovocytes (cas d'abandon de la première cellule) ou par un “ état d'urgence » qui serait un excès de maturation (cas de la ponte sur une provision étrangère ou de la ponte de 2 œufs sur la même provision). Seul un (c état de besoin ) normal pousse la femelle à accomplir toute la séquence de nidification.

\section{CONCLUSION GÉNÉRALE}

Les traits de comportement communs aux deux espèces sont les suivants :

- Elles nidifient de préférence sur les façades de bâtiments de pierre ou de bois, exposées au nord ou à l'ouest.

- Les nids ont une structure linéaire et les cloisons sont en boue séchée.

- L'approvisionnement d'une cellule s'effectue en serre en 4-5 heures.

- Le comportement de butinage dans les conditions naturelles est du type polylectique. Les deux espèces manifestent cependant une tendance à l'oligolectie. Les femelles récoltent le pollen sur 8 à 9 familles botaniques (une douzaine d'espèces de plantes) 8 espèces sont butinées par $O$. cornuta et $O$. rufa : Prunus sp., Pirus communis, Pirus malus, Crataegus sp., Quercus pedunculata, Ranunculus bulbosus, Brassica napus oleifera, Ilex aquifolium. 
Chaque espèce a des traits de comportement qui la différencient de l'autre :

- Lors de la construction des cellules, $O$. rufa seule édifie un « pas de porte ». La distribution des longueurs de cellules est plus étalée chez $O$. cornuta. En moyenne une cellule d'O. cornuta est plus longue qu'une cellule d'O. rufa.

- O. cornuta est moins polylectique qu'O. rufa. La première espèce récolte surtout du pollen de Prunus, la seconde le pollen de Quercus et Ranunculus.

- Les provisions d'O. rufa ont un aspect pulvérulent, alors qu'elles sont humides chez $O$. cornuta. Les œufs d' $O$. rufa sont saupoudrés de pollen. Ceux d'O. cornuta sont absolument propres.

L'étude comparative met en évidence des caractères permettant de discriminer deux espèces toujours considérées comme très voisines.

La lecture des spectres de la récolte de pollen laisse supposer qu'O. cornuta est un agent pollinisateur intéressant pour les espèces de Rosacées précoces. $O$. rufa, très attirée par les pollens de plantes non cultivées semble moins importante sur le plan agronomique.

Les commodités procurées par l'élevage en serre devraient permettre une analyse expérimentale plus complète du comportement de nidification.

Reçu pour publication en avril 1973.

Eingegangen im April 1973.

\section{ZUSAMMENFASSUNG}

Osmia carnuta und Osmia rufa, zwei nicht nur in Frankreich sondern in ganz Europa allgemein vorkommende Arten, sind Gegenstand vergleichender Untersuchungen. Während zweier aufeinanderfolgender Jahre wurde das Verhalten beim Bau, bei der Ausstattung der Nester und beim Sammeln von Vorräten in Lusignan (Frankreich) untersucht und zwar unter natürlichen Bedingungen und im geschlossenen Raum (Gewächshaus).

Die Beobachtungsanordnung in der freien Natur ist die gleiche, wie sie in einer früheren Arbeit (TASEI, 1972) beschrieben wurde. Es handelt sich dabei um sogen. Fallennester mit der Länge nach gespaltenen Rohrstengeln von verschiedenem Durchmesser. Die Zucht im Gewächshaus beginnt mit im Freien gefangenen Weibchen, die zu bauen begonnen haben. Die Mikroanalyse der Pollenvorräte erfolgte nach der Methode von Maurizio-Louveaux (1965).

\section{Ergebnisse}

1. Abbildung 1 und Tabelle 2 zeigen, dass die Flug- und Nestbau- Perioden für $O$. cornuta von Anfang April bis Anfang Juni liegen, die für O. rufa von Ende April bis Ende Juni. Über die Tätigkeit im Gewächshaus gibt Tab. 3 Auskunft.

2. Die Architektur des Nestes (Abb. 2) ist seit langem bekannt. Unsere Messungen ergaben grosse Unterschiede in der Zellenlänge bei 0 . cornuta $: 12,5 \mathrm{~mm}$ bis $18 \mathrm{~mm}$ und geringe bei O. rufa : $10 \mathrm{~mm}$ bis $12 \mathrm{~mm}$ je nach Durchmesser (Tab. 4). Das Verhalten von $O$. rufa beim Bau des Nestes unterscheidet sich wesentlich von dem von 0 . cornuta. O. rufa legt im Verlauf 
der Nestausstattung fast immer Schwellen vor den Zelleneingängen an, ganz im Gegensatz zu $O$. cornuta. Unter natürlichen Bedingungen wählen die Weibchen für ihre Nistplätze Löcher in altem Gebälk oder Gemäuer. Auffallend ist die Bevorzugung der Nord- und Westlage (Tab. 6 u. 7).

3. Die Versorgung einer Zelle (Tab. 14) erfordert im Gewächshaus $41 / 2$ bis 5 Stunden Sammeltätigkeit bei beiden Arten. Diese Zeit entspricht 35-40 Sammelfügen bei 0 . cornuta und 30 bei $O$. rufa. Durchschnittlich dauert ein Sammelflug bei $O$. cornuta 5-6 Min und 6-9 Min bei $O$. rufa. Bei beiden Arten wechseln Serien kurzer Aus flüge (1-4 Min) mit länger dauernden (8-17 Min) ab.

Die Vorräte von $O$. rufa sowie ihre Eier werden mit trockenem Pollen eingepudert, während die Vorräte von $O$. cornuta gleichmässig feucht und fest sind und die Eier frei von Pollen.

4. Die Beobachtungen im geschlossenen Raum (Gewächshaus) (Tab. 9-13) zeigen der Pflanzen

- die Bedeutung der Untersuchungen für die Feststellung der relativen Anziehungskraft

- die Polylektie (Sammeln einer Vielfalt von Pollenarten) beider Osmienarten

- das gleiche Verhalten in bezug auf Rosaceen, Cruciferen, Salicaceen und Papilionaceen

- das verschieden Verhalten der beiden Weibchen von $O$. rufa.

5. Unter natürlichen Bedingungen sammelt $O$. cornuta Pollen von elf botanischen Arten aus acht Familien : Rosaceen, Liliaceen, Kompositen, Fagaceen, Ranunculaceen, Cruciferen, Aquifoliaceen, Papaveraceen, Cistaceen, Caryophyllaceen, Papilionaceen, (52 analysierte Vorräte) s. Tab. 15. Abb. 3, angefertigt nach der in Tab. 1 beschriebenen Methode, beweist, dass bei beiden Osmien-Arten die Tendenz besteht, sich oligolektisch (d. h. sich auf einige wenige Pollenarten zu beschränken) zu verhalten, was bei $O$. cornuta stärker ausgeprägt ist. (Tab. 12). Bei $O$. rufa kommt es indessen vor, dass mehr Ranunculus-Pollen als Quercus-Pollen gesammelt wird. Aus Tab. 18 wird ersichtlich, dass die Pollenernte mehr oder weniger konstant ist. Prunus, Pirus, Crataegus werden während der gesamten Blütezeit be flogen, Ranunculus und Papaver nur in der Hauptblütezeit. Gewisse Pflanzen wie Helianthemum, Ilex, Tulipa, Vicia und Stellaria werden nur gelegentlich beflogen. Im Spektrum der Pollenernte zeigt sich zweifellos das Zusammenwirken zweier Tendenzen : der ererbten Tendenz, einige P flanzen häufiger als andere zu befliegen und die Tendenz, solche $P$ flanzen zu besuchen, die in grosser Zahl vorhanden sind.

6. Eine kurze Analyse des Nestbau-Verhaltens beweist, dass beide Arten künstliche Bauten entfernen, wenn sie die normale Weiterentwicklung der Nestanlage behindern. Wenn der Beobachter eine im Bau befindliche Anlage oder deren Ausstattung vervollständigt, kommt es vor, dass das Weibchen diese fremden Zugaben zurückweist, es kann aber auch die neue Situation annehmen und die Arbeit des Beobachters zu Ende führen. Die Zerstörung einer Scheidewand während der Bauarbeiten veranlasst das Weibchen, das Mauern wieder aufzunehmen. Nach der Eiablage werden die Entfernung des Eies und der Vorräte nicht mehr beachtet.

\section{Schlussfolgerungen}

Das vergleichende Studium von Osmia cornuta und Osmia rufa lässt deutlich gewisse Eigenschaften erkennen, die es erlauben, diese beiden Arten, die bisher für einander sehr nahestehend galten, zu unterscheiden. Ausserdem lassen die Pollenspektren vermuten, dass o. cornuta für die Bestäubung frühblühender Rosaceen von Bedeutung ist.

\section{REMERCIEMENTS}

Nous remercions M. Lecomte pour ses conseils au cours de cette étude, ainsi que M. Louveaux pour l'aide qu'il nous a apportée dans la détermination des pollens. 


\section{RÉFÉRENCES BIBLIOGRAPHIQUES}

Bonelli B., 1966. Osservazioni biologiche sugli imenotteri melliferi e prédatori della val di Fieume. Osmia rufa L.. Studi trent. Sci. nat., 43, 1, 46-56.

Descy A., 1968. Observations et expériences en vue de modifier le comportement de certains Hyménoptères. Bull. Biol., 102, 4, 391-431.

FABre J.-H., 1933. Souvenirs entomologiques, $3^{\mathrm{e}}$ série. Etude sur l'instinct et les mceurs des Insectes. Delagrave, Paris.

Ferton C., 1923, La vie des abeilles et des guêpes. Chiron, Paris.

Free J.-B., Wiltrams I.-H., 1970, Preliminary investigations on the occupation of artificial nests by Osmia rufa L. (Hymn. Megachilidae). J. appl. Ecol., 7, 559-566.

Friese H., 1923, Die europäischen Bienen (Apidae). Das Leben und Wirken unserer Blumenwespen. De Gruyter, Berlin u. Leipzig.

Hardouin R., 1948, La vie des abeilles solitaires. $4^{\mathrm{e}}$ éd. Gallimard, Paris.

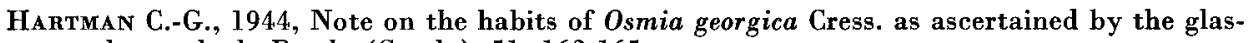
stube method. Psyche (Camb.), 51, 162-165.

Héroin-Delaunay Y., 1966, Étude des constructions d'Osmia cornuta (Hym. Apoïdea) après injection de produits marqués. $C$. $R$. Soc. Biol., 160, 2, 2027-2030.

IugA V.-G., 1962, The Apoïds as pollinators. Trav. Mus. Hist. nat. « gr. Antipa », 3, 225-237.

Leсомте J., 1963-1965, Quelques observations sur la biologie des Osmies (non publié).

LEVIN M.-D., 1966, Biological notes on Osmia lignaria and Osmia californica. J. Kansas entomol. Soc., 39, 3, 524-535.

LiNSLEY E.-G., 1958, The ecology of solitary bees. Hilgardia, 27, 543-599.

LosıNski P., 1936, Über einen eigentümlichen Nestbau von Osmia bicornis L. Z. wiss. Insektenbiol., 7, 223-230 et 316-322.

Malyshev S.-I., 1935, The nesting habits of solitarybees. A comparative study. Eos. XI. Lams III-XV.

MALYSheva M.-S., 1958, Leaf cutter bees of the genus Megachile latr. (Hymenoptera, Megachilidae) infesting young forest plantations in the U.S.S.R. Entomol. Obozrenie, 37, 2, 319-329.

Maréchal P., 1926, Étude biologique d'Osmia aurulenta Panz. Bull. biol. Fr. Belg., 60, 561592.

Manikovskaya T. P., 1968, New data on the biology of some species of social bees (Hymenoptera, Megachilidae) from Southeast Kazakhstan. Entomol. Rev., 47, 4, 487-491.

Maurizio A., Louveaux J., 1965, Pollens de plantes mellifères d'Europe. Union des groupements apicoles français, Paris.

Mryamoto S., 1962, Outline of flower relationships of Japonese bees. Acta Hymenopterologica $1,393-455$.

Popovicr Baznosanu A., 1909, Étude biologique comparative de quelques espèces d'Osmia. Arch. Zool. exper. gen., 5, 2, 1-26.

Popovici Baznosand A., 1910, Relation entre la taille de l'adulte et la quantité de nourriture absorbée par les larves chez Osmia rufa et Osmia cornuta. C.R. Soc. Biol., 68, 480-481.

Robertson C., 1925, Heterotropic bees. Ecology, 6, 412-436.

SAZ R. P. E., 1929, Nido de Himenoptero en tubo de vidrio. Bol. Soc. Iber. Zaragoza, 29, 132-133.

TASEI J.-N., 1972, Observations préliminaires sur la biologie de Osmia coerulescens L. pollinisateur de la luzerne. Apidologie, 3, 149-165. 